\title{
Glycogen synthase kinase-3 $\beta$ mediates convergence of protection signaling to inhibit the mitochondrial permeability transition pore
}

\author{
Magdalena Juhaszova, ${ }^{1}$ Dmitry B. Zorov, ${ }^{1,2}$ Suhn-Hee Kim, ${ }^{1,3}$ Salvatore Pepe, ${ }^{1,4}$ Qin Fu, ${ }^{1}$ \\ Kenneth W. Fishbein, ${ }^{5}$ Bruce D. Ziman, ${ }^{1}$ Su Wang, ${ }^{1}$ Kirsti Ytrehus, ${ }^{1,6}$ Christopher L. Antos, ${ }^{7}$ \\ Eric N. Olson, ${ }^{7}$ and Steven J. Sollott ${ }^{1}$
}

\begin{abstract}
1 Laboratory of Cardiovascular Science, Gerontology Research Center, Intramural Research Program, National Institute on Aging, NIH, Baltimore, Maryland, USA. ${ }^{2}$ Department of Bioenergetics, A.N. Belozersky Institute of Physico-Chemical Biology, Moscow, Russia. ${ }^{3}$ Department of Physiology,

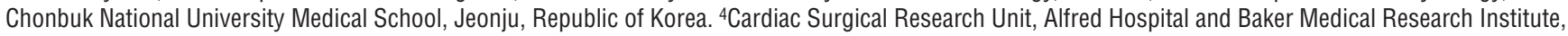
Monash University Faculty of Medicine, Melbourne, Australia. 5Laboratory of Clinical Investigation, Gerontology Research Center, Intramural Research Program, National Institute on Aging, NIH, Baltimore, Maryland, USA. ${ }^{6}$ Department of Medical Physiology, Institute of Medical Biology, University of Tromsø, Tromsø, Norway. ${ }^{7}$ Department of Molecular Biology, University of Texas Southwestern Medical Center at Dallas, Dallas, Texas, USA.
\end{abstract}

\begin{abstract}
Environmental stresses converge on the mitochondria that can trigger or inhibit cell death. Excitable, postmitotic cells, in response to sublethal noxious stress, engage mechanisms that afford protection from subsequent insults. We show that reoxygenation after prolonged hypoxia reduces the reactive oxygen species (ROS) threshold for the mitochondrial permeability transition (MPT) in cardiomyocytes and that cell survival is steeply negatively correlated with the fraction of depolarized mitochondria. Cell protection that exhibits a memory (preconditioning) results from triggered mitochondrial swelling that causes enhanced substrate oxidation and ROS production, leading to redox activation of PKC, which inhibits glycogen synthase kinase-3 $\beta$ (GSK-3 $\beta$ ). Alternatively, receptor tyrosine kinase or certain $G$ protein-coupled receptor activation elicits cell protection (without mitochondrial swelling or durable memory) by inhibiting GSK-3 $\beta$, via protein kinase B/Akt and $\mathrm{mTOR} / \mathrm{p}^{70^{\mathrm{sk}}}$ pathways, PKC pathways, or protein kinase A pathways. The convergence of these pathways via inhibition of GSK- $3 \beta$ on the end effector, the permeability transition pore complex, to limit MPT induction is the general mechanism of cardiomyocyte protection.
\end{abstract}

\section{Introduction}

Convergence of molecular signaling provides powerful integration and control of the system nearby the terminal molecular target. The mitochondria have such critical signaling targets (1), thus providing the opportunity for precisely regulated single-step control points. Controlling the balance between survival and death signaling, where mitochondria play a key role, determines cell fate. Understanding of these survival and death signals is required to devise measures to limit pathological damage to tissues composed of postmitotic cells such as the heart and brain.

Among all factors that cause unwanted cell death, periods of prolonged hypoxia followed by reoxygenation cause some of the most damaging and irreversible consequences. The most potent form of protection capable of reducing cell death following prolonged periods of ischemia (as would accompany arterial occlu-

Nonstandard abbreviations used: bisindolylmaleimide I (BIS); 2-chloro-N6-cyclopentyladenosine (CCPA); diazoxide (Dz); 2',7'-dichlorodihydrofluorescein diacetate (DCF); glucagon-like peptide-a (GLP-1); glycogen synthase kinase-3 (GSK-3); 5-hydroxydecanoate (5HD); indanyloxyacetic acid 94 (IAA94); mitochondrial ATPdependent $\mathrm{K}^{+}$channel (mitoK $\mathrm{K}_{\mathrm{ATP}}$ ); mitochondrial permeability transition (MPT); $\mathrm{N}$-acetyl-L-cysteine (NAC); partial fatty acid oxidation (PFAO); $\mathrm{Na} / \mathrm{H}$ exchange (NHE); preconditioning (PC); protein kinase $\mathrm{A}(\mathrm{PKA})$; protein kinase $\mathrm{B}(\mathrm{PKB})$; reactive oxygen species (ROS); receptor for activated C kinase (RACK); Sanglifehrin A (SFA); short interfering RNA (siRNA); S-nitroso- $N$-acetyl-penicillamine (SNAP); tetramethylrhodamine methyl ester (TMRM); transgenic (TG); transmembrane potential $(\Delta \Psi)$; trimetazidine (TMZ); Tyr-D-Ala-Gly-Phe-D-Leu (DADLE).

Conflict of interest: The authors have declared that no conflict of interest exists.

Citation for this article: $J$. Clin. Invest. 113:1535-1549 (2004).

doi:10.1172/JCI200419906. sion) results from the activation of endogenous mechanisms triggered by brief episodes of transient ischemia and reperfusion preceding the prolonged insult (ref. 2; reviewed in ref. 3). This phenomenon is known as ischemic preconditioning. The full set of mechanisms in preconditioning (PC) is not clear, and the current paradigm implicates the activation of one or more $G$ protein-coupled receptors by adenosine, bradykinin, or opioids, followed by a cascade of protein kinases, including PKC and MAPK, which either leads to, or is a consequence of, activation of the mitochondrial ATP-dependent $\mathrm{K}^{+}$channel (mitoK $\mathrm{K}_{\text {ATP }}$ ) (4-6) and reactive oxygen species (ROS) production $(7,8)$. Various pharmacologic agents can mimic PC and have been proven to be cardio/ neuroprotective in experimental models.

PC and pharmacologic activation of the mito $_{\text {ATP }}$ inhibit oxidative stress-induced apoptosis in cardiac myocytes (9). However, the molecular mechanisms by which activation of the mitoK $\mathrm{K}_{\text {ATP }}$ protects against apoptosis, and, more generally, the end effector(s) of protection, remain a matter of debate. There is increasing evidence that the mitochondrial permeability transition (MPT) (10), which plays a central role in mitochondria-mediated death pathways (1), occurs in heart as a result of ischemia/reperfusion injury (11-13), and recent data suggest that this could be involved in the mechanism of protection by the mitoK ${ }_{\text {ATP }}(9,14-16)$. We have previously developed a model enabling the precise determination of the MPT sensitivity to oxidant stress in intact cardiac myocytes (17). Using this technique, we demonstrate here that hypoxia/reoxygenation significantly reduces the ROS threshold for the MPT, that cardiac myocyte survival is steeply negatively correlated with the fraction 
of depolarized mitochondria, and that PC and cardio/neuroprotective agents acting via distinct mechanisms all promote cell survival by limiting MPT induction. We find that a diversity of upstream signaling pathways (including protein kinase A [PKA], $\mathrm{PKB}, \mathrm{PKC}$, and $\left.\mathrm{p} 70^{\mathrm{s} 6 \mathrm{~K}}\right)$ all appear to converge to cause a similar degree of functional protection of the permeability transition pore complex (the end effector), which suggests that a point of integration on a master switch immediately proximal to the permeability transition pore complex could be involved.

We identify glycogen synthase kinase-3 (GSK-3) as the pivotal kinase that serves as this point of protection-signaling integration, as it is known to receive inputs from each of these pathways, which in turn regulate its enzymatic activity. Indeed, a recent report found that pharmacologic inhibition of GSK-3 reduced infarct size and improved postischemic function (18). There are two GSK- 3 isoforms, $\alpha$ and $\beta$ (51 and $47 \mathrm{kDa}$, respectively), which have $98 \%$ identity in their central $30-\mathrm{kDa}$ catalytic domain (19). These isoforms can exhibit different catalytic activities toward a number of intracellular substrates, with a general trend for the $\beta$ isoform to have a comparatively higher activity versus the $\alpha$ (20). Although both isoforms may subserve different cellular functions, a certain amount of functional redundancy could still occur. However, deletion of the GSK-3 $\beta$ gene results in developmental arrest and embryonic lethality, thus showing that endogenous GSK-3 $\alpha$ signaling is unable to compensate and rescue the GSK-3 $\beta$-null mice (21).

We use RNA interference to prove that protection signaling operates via the $\beta$ rather than the $\alpha$ isoform of GSK-3 in cardiac myocytes. GSK-3 $\beta$ is highly active in unstimulated cells and becomes inactivated in response to mitogenic stimulation (reviewed in ref. 22). Inactivation of GSK-3 $\beta$ by site-specific phosphorylation usually results in activation of downstream signaling pathways (23). The activity of GSK-3 $\beta$ is inversely related to the phosphorylation status of serine-9. Dephosphorylation of this site, or mutations that prevent phosphorylation, result in activation of the kinase. Based on these properties of the enzyme, we confirm that protection signaling requires GSK-3 $\beta$, using a transgenic mouse model with cardiac-specific expression of a constitutively active (signalresistant) form of GSK-3 $\beta$ containing a serine-9-to-alanine mutation (24). Finally, we demonstrate that the mitoK $_{\text {ATP }}$ is but one of many possible mechanisms leading to protection, and we conclude that the general mechanism of protection is the convergence of these pathways via inhibition of the master switch kinase, GSK-3 $\beta$, on the end effector, the permeability transition pore complex, to limit MPT induction.

\section{Methods}

Cells. Adult cardiac myocytes were isolated from Sprague-Dawley rats (2-4 months old), and WT or transgenic (TG) mice expressing a signal-resistant form, GSK-3 $\beta$ S9A, by standard enzymatic techniques (25). Cells were suspended in a solution containing (in $\mathrm{mM}): \mathrm{NaCl} 137, \mathrm{KCl} 4.9, \mathrm{MgSO}_{4} 1.2, \mathrm{NaH}_{2} \mathrm{PO}_{4} 1.2$, glucose 15, HEPES 20, and $\mathrm{CaCl}_{2} 1.0$ (pH 7.4).

Mitochondria were isolated by differential centrifugation of the heart homogenate.

Neonatal cardiac myocytes were isolated from 1- to 3-day-old Wistar rats by enzymatic digestion as outlined previously (26). Culture media and additives were purchased from Invitrogen Corp. (Carlsbad, California, USA). Isolated myocytes were resuspended in media consisting of four parts high-glucose DMEM, one part Medium 199 (Invitrogen Corp.) 10\% horse serum, 5\% FBS, 2 mM
L-glutamine, penicillin $(100 \mathrm{U} / \mathrm{ml})$ and streptomycin $(100 \mu \mathrm{g} / \mathrm{ml})$ and filtered through a sterile $70-\mu \mathrm{m}$ cell strainer to remove debris. The myocytes were then preplated on uncoated Falcon 100-mm tissue-culture dishes (BD Biosciences, San Jose, California, USA) for 45 minutes to allow selective attachment of nonmyocytes to the dishes. Myocytes were then seeded at a density of $6.0 \times 10^{4} \mathrm{cells} / \mathrm{cm}^{2}$ on 35-mm glass-bottom dishes (MatTek Corp., Ashland, Massachusetts, USA) precoated with $10 \mu \mathrm{g} / \mathrm{ml}$ bovine plasma fibronectin.

Handling of animals and experimental procedures were conducted in accordance with NIH guidelines for animal care and use.

GSK-3 gene silencing through RNA interference. Second-day cultured neonatal cardiac myocytes were transfected with a pool of four short interfering RNAs (siRNAs; $100 \mathrm{nM}$ ) targeted specifically to GSK- $3 \alpha$ and to GSK-3 $\beta$ using GeneSilencer reagent (Gene Therapy Systems Inc., San Diego, California, USA) according to the protocol provided by the company. SiRNA duplexes were designed and synthesized by Dharmacon Inc. (Lafayette, Colorado, USA) to target the rat GSK-3 $\alpha$ : (a) 5'-CGACAAAGGTGTTCAAATC-3', (b) 5'-GATCATCCCTATCATCTAT-3', (c) 5'-GAGCAAATCCGAGAGATGA$3^{\prime}$, and (d) 5'-AGAAAGATGAGCTGTATTT-3'; and the rat GSK-3 $\beta$ : (a) 5'-GATCTGCCATCGAGACATT-3', (b) 5'-CTCAAGAACTGTCAAGTAA-3', (c) 5'-TCAGAAGTCTAGCCTATAT-3', and (d) 5'ACACTAAAGTCATTGGAAA- $3^{\prime}$. As a negative control, cells were transfected with siRNA against GFP (Dharmacon Inc.). Experiments were performed 72 hours after transfection.

Cell treatment. The following compounds were applied (alone or in combination) to cells during experimental measurements: 2 nM IGF-1 (Upstate Biotechnology Inc., Lake Placid, New York, USA); $30 \mathrm{nM}$ insulin; $10 \mathrm{nM}$ leptin; $10 \mathrm{nM}$ glucagon-like peptide-1 (GLP-1); 1 nM rapamycin; $10 \mu \mathrm{M}$ LY 294002; 50 nM wortmannin; 100 nM PMA; 100 nM bisindolylmaleimide I (BIS); 3 mM LiCl (Calbiochem-Novabiochem Corp., San Diego, California, USA); $0.1 \mathrm{mM}$ Rp-8-CPT-cAMPS (BIOLOG Inc., Hayward, California, USA); $3 \mu \mathrm{M}$ SB 216763 and $30 \mu \mathrm{M}$ SB 415286 (Tocris Cookson Inc., Ellisville, Missouri, USA); $1 \mu \mathrm{M}$ Sanglifehrin A (SFA; Novartis, Basel, Switzerland); $400 \mathrm{nM}$ bradykinin; $200 \mathrm{nM}$ 2-chloroN6-cyclopentyladenosine (CCPA); $200 \mathrm{nM}$ cyclosporin A; $10 \mathrm{nM}$ Tyr-D-Ala-Gly-Phe-D-Leu (DADLE); $30 \mu \mathrm{M}$ diazoxide (Dz); $50 \mu \mathrm{M}$ pinacidil; $500 \mu \mathrm{M}$ 5-hydroxydecanoate (5HD); $10 \mu \mathrm{M}$ Hoe694; 10 $\mu \mathrm{M}$ indanyloxyacetic acid 94 (IAA94); $1 \mu \mathrm{M}$ trimetazidine (TMZ); $0.5 \mathrm{mM}$ palmitic acid; $0.5 \mathrm{mM}$ octanoic acid; and $1 \mathrm{mM} \mathrm{N}$-acetyl-Lcysteine (NAC; Sigma-Aldrich, St. Louis, Missouri, USA).

Confocal microscopy and determination of MPT ROS threshold. Cardiac myocytes were loaded with $125 \mathrm{nM}$ tetramethylrhodamine methyl ester (TMRM) (Molecular Probes Inc., Eugene, Oregon, USA) for more than 2 hours at room temperature and imaged with an LSM 510 inverted confocal microscope (Carl Zeiss Inc., Jena, Germany). In certain protocols cells were coloaded with $10 \mu \mathrm{M} 2^{\prime}, 7^{\prime}$-dichlorodihydrofluorescein diacetate (DCF; Molecular Probes Inc.) for 15 minutes at room temperature. Time scans at $2 \mathrm{~Hz}$ were recorded from mitochondria arrayed along individual myofibrils in adult myocytes (and along protofibrillar clusters in neonatal myocytes) in a multichannel line-scan mode as appropriate for the dyes loaded with excitation at $488 \mathrm{~nm}$ (for DCF) and $543 \mathrm{~nm}$ (for TMRM), collecting emission at 500-530 $\mathrm{nm}$ and greater than $560 \mathrm{~nm}$, respectively. Images were processed by MetaMorph software (Universal Imaging Corp., Downingtown, Pennsylvania, USA). MPT ROS threshold $\left(t_{\mathrm{MPT}}\right)$ was measured as the average time necessary to induce MPT in a row consisting of 25 mitochondria. Experiments were carried out at $23^{\circ} \mathrm{C}$ except where indicated otherwise. 
A

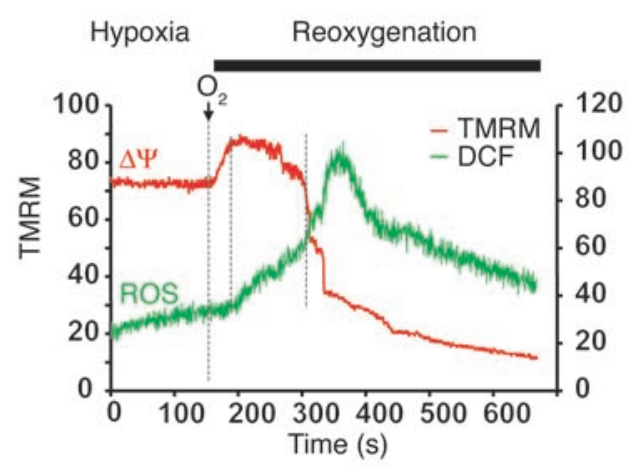

B
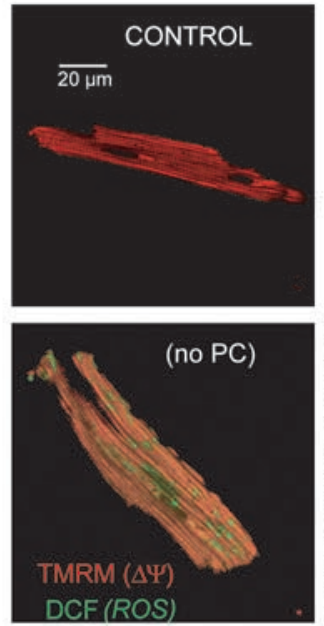

Hypoxia/Reoxygenation
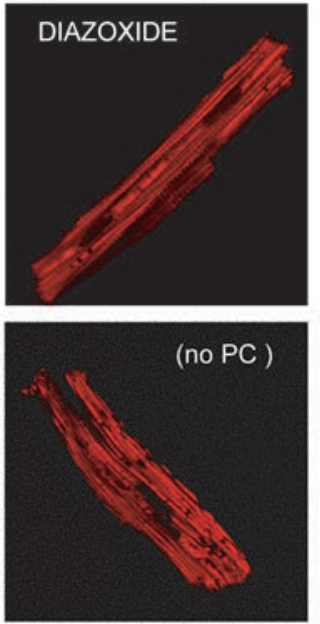

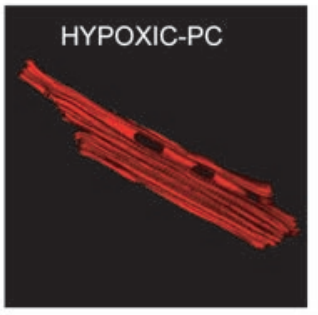

HYPOXIC-PC + 5HD

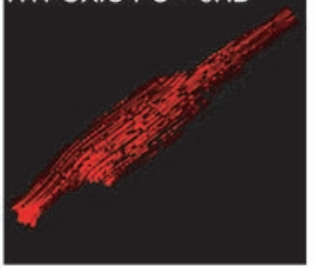

C

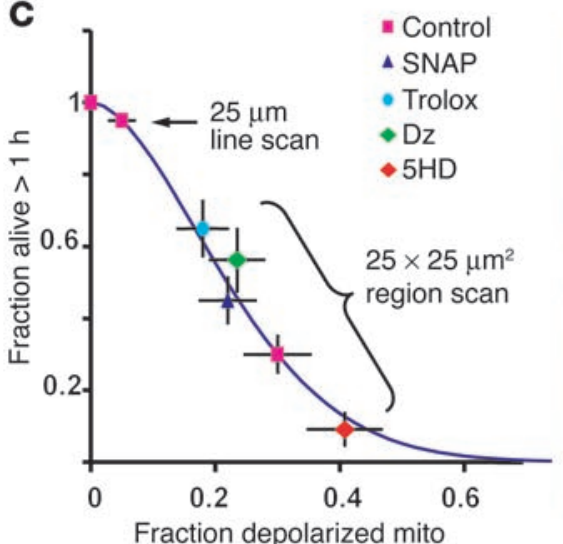

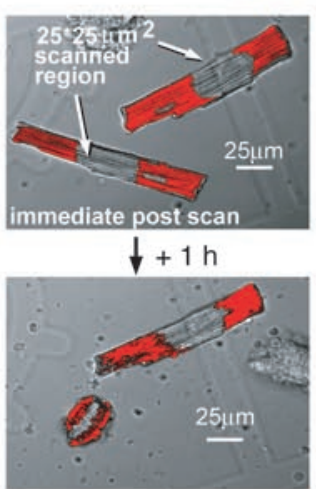

D

E

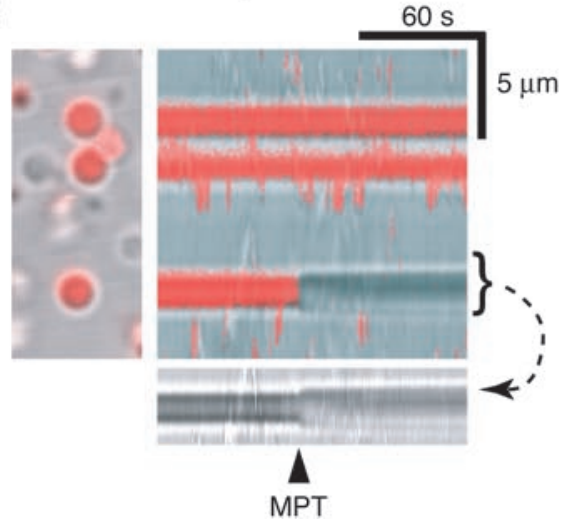

Figure 1

ROS are involved in mitochondrial deterioration during hypoxia/reoxygenation. (A) Reoxygenation-induced mitochondrial hyperpolarization leads to increased ROS. Mitochondria stained with $\operatorname{TMRM}(\Delta \Psi$, red) and DCF (ROS, green) were laser line-scanned (2 Hz) during hypoxia and the reoxygenation phase. The ROS burst is delayed after reoxygenation and starts at the maximum $\Delta \Psi$. Mitochondrial hyperpolarization lasts for approximately 2 minutes, followed by loss of $\Delta \Psi$. (B) $\Delta \Psi$ loss in a significant fraction of mitochondria, caused by hypoxia/reoxygenation. Depolarized mitochondria (red-fluorescence "holes"; bottom panels) are associated with increased ROS (green; bottom left panel). Hypoxic PC or pharmacologic PC (represented by Dz) prevents mitochondrial depolarization, and $5 \mathrm{HD}$ accentuates the loss. (C) Cell survival after constant-energy photoexcitation of a $25 \times 25 \mu \mathrm{m}^{2}$ region. The right panels show TMRM-stained cells (red) immediately after, and 1 hour after, irradiation. Survival is inversely related to the fraction of mitochondria (mito) undergoing MPT induction and is improved by ROS scavenger (Trolox), NO donor (SNAP), and Dz and impaired by 5HD. (D) Methodology used to determine the ROS threshold of MPT induction. Mitochondria stained with TMRM (red) were laser line-scanned until MPT induction. The average time required for the standardized photoproduction of ROS to cause MPT induction $\left(t_{\mathrm{MPT}}\right)$ is taken as the index of the ROS threshold in that cell. $(\mathrm{E})$ Two-hertz line scan of individual isolated cardiac mitochondria. Light transmittance (gray) and TMRM fluorescence (red) are overlaid. The abrupt loss of $\Delta \Psi$ (TMRM) and increase in volume (arrow) are similar to those observed in situ (D). Vertical flickering in the image is an artifact caused by movement of adjacent floating mitochondria. 

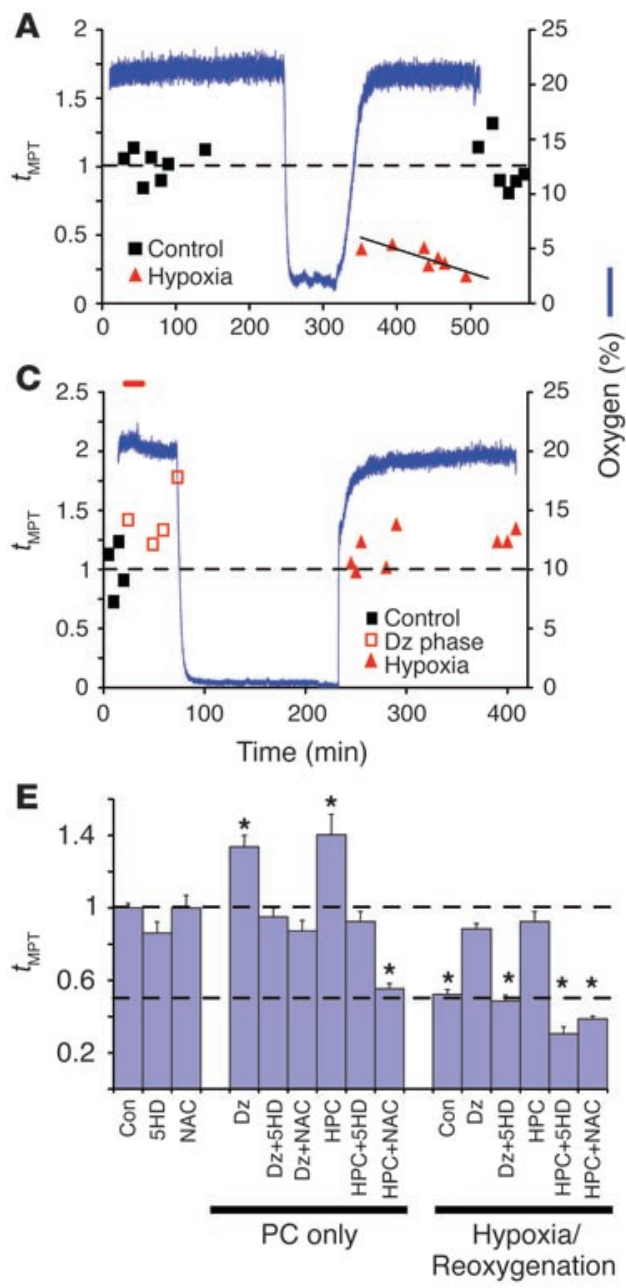
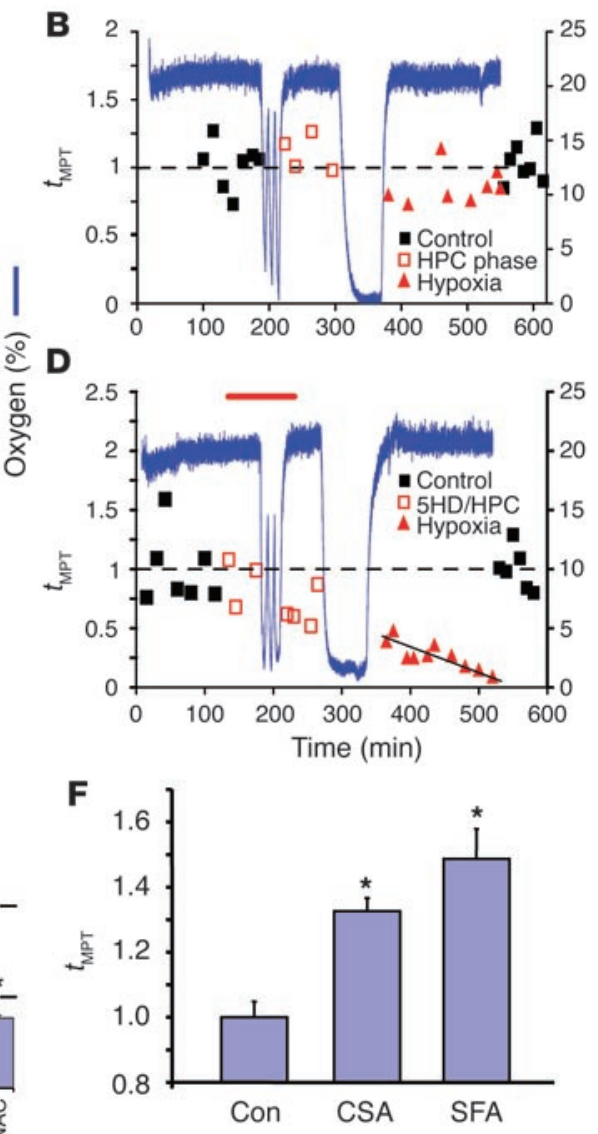

\section{Figure 2}

Change in MPT ROS threshold $\left(t_{\mathrm{MPT}}\right)$ under reoxygenation and hypoxic and pharmacologic PC. (A) Hypoxia/reoxygenation causes a rapid and progressive decline in $t_{\mathrm{MPT}}$ (control, black squares; after reoxygenation, red triangles; $\mathrm{O}_{2}$ in the buffer, blue trace). (B) Hypoxic (HPC) (three 5-minute cycles of hypoxia/reoxygenation) improves $t_{\text {MPT }}$ (red triangles compared with control, shown in black squares). (C) Dz pretreatment (30 $\mu \mathrm{M}$ for 15 minutes, red bar) prevents the decline in $t_{\mathrm{MPT}}$ after hypoxia/reoxygenation. (D) $5 \mathrm{HD}$ treatment (500 $\mu \mathrm{M}$, during hypoxic PC phase only, red bar) abolishes the hypoxic PC-mediated protection against the decline in $t_{\mathrm{MPT}}$ after hypoxia/reoxygenation. (E) Summary of the effects of hypoxia/reoxygenation and hypoxic and pharmacologic PC on $t_{\mathrm{MPT}}$. ${ }^{\star} P<0.01$ vs. control (Con). (F) Both cyclosporin A (CSA) $(0.2 \mu \mathrm{M})$ and SFA $(1 \mu \mathrm{M})$ enhance MPT ROS threshold (measured more than 60 minutes after washout). ${ }^{*} P<0.01$ vs. control.
Transmitted optics line-scan imaging (14.1 pixels/ $\mu \mathrm{m}$ along the long axis of the cell for 72.6 or $145.1 \mu \mathrm{m}$ ) was performed to assess changes in mitochondrial volume. Fourier analysis (ImageJ, W. Rasband, NIH, Bethesda, Maryland, USA) of repeating intensity provided the long-axis spacing of the sarcomere and mitochondrial compartments (from the first- and second-order spectral peaks, enabling resolution of changes in dimension of $\sim 1 \%$ in 1 $\mu \mathrm{m}$ structures; see Supplement 1 for further details and validation of method; all supplemental material available at http://www.jci. org/cgi/content/full/113/11/1535/DC1). Spatial information from P-GSK-3 $\beta$ (Cell Signaling Technology Inc., Beverly, Massachusetts, USA) immunofluorescence images was analyzed by two-dimensional Fourier transform (ImageJ) to assess the degree of signal compartmentalization. The spectral power of the higherorder components in the frequency domain was normalized to the zero-order peak and taken as the index of compartmentalization.

Hypoxia and $\mathrm{VO}_{2}$ experiments. Myocytes were placed into a sealed microscope chamber connected to a pump providing rapid changes of superfusate buffers of defined $\mathrm{pO}_{2}$ (saturated with moisturized $100 \% \mathrm{~N}_{2}$ or $21 \% \mathrm{O}_{2}$ air) and maintained at $37^{\circ} \mathrm{C}$. Oxygen in the chamber was measured using a fiberoptic oxygen sensor combined with a CHEM2000 spectrometer (Ocean Optics Inc., Dunedin, Florida, USA). Cell respiration $\left(\mathrm{VO}_{2}\right)$ was measured by a Clark-type $\mathrm{O}_{2}$ electrode coupled to a polarograph. Cell death was determined by morphologic criteria (cell rounding) together with positive staining with propidium iodide. Rare rod-shaped myocytes that appeared weakly stained by propidium iodide (numerically insignificant at $<<1 \%$ of population), probably representing cell ghosts, were counted as dead cells.

Immunolabeling. Myocytes were exposed to protective treatments and appropriate inhibitors, and then either cells were fixed with $2 \%$ formaldehyde, or soluble and particulate cell fractions or cell lysates were prepared. Immunoblotting and immunocytochemistry were performed according to published protocols (Cell Signaling Technology Inc.). Antibodies used were $\varepsilon$ PKC (Research \& Diagnostic Antibodies, Benicia, California, USA), GSK-3 $\beta$ (Santa Cruz Biotechnology Inc., Santa Cruz, California, USA; and Cell Signaling Technology Inc.), P-GSK-3 $\beta$ (Cell Signaling Technology Inc.), and $39-\mathrm{kDa}$ subunit of the mitochondrial Complex I (Molecular Probes Inc.).

Electron microscopy. Hearts were perfused with $4 \%$ paraformaldehyde and $0.05 \%$ glutaraldehyde, cut into pieces, incubated with antibodies against PKC (Research \& Diagnostics Antibodies) and then with gold-conjugated secondary antibodies, fixed with $2 \%$ glutaraldehyde, postfixed with $\mathrm{OsO}_{4}$, dehydrated, and embedded into Epon. Ultrathin sections were viewed by a Hitachi HU-11 (Hitachi Ltd., Tokyo, Japan) electron microscope.

Statistics. All experiments were performed at least in triplicate, with cell number greater than 12 in each independent experiment unless stated otherwise. All data are mean \pm SEM. Comparisons 
A

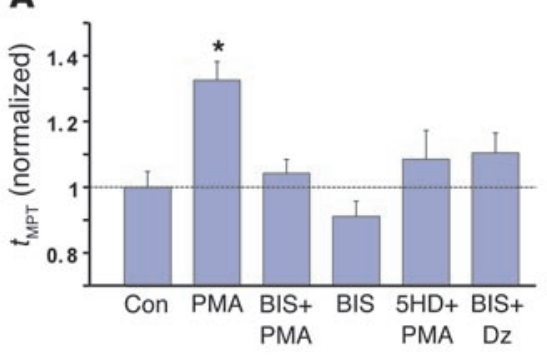

C

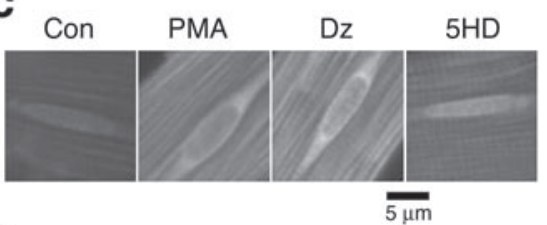

D
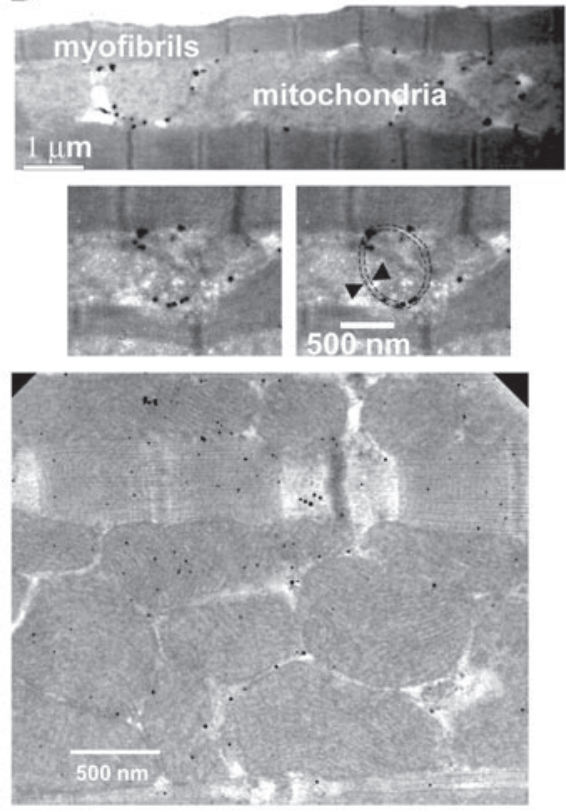

B

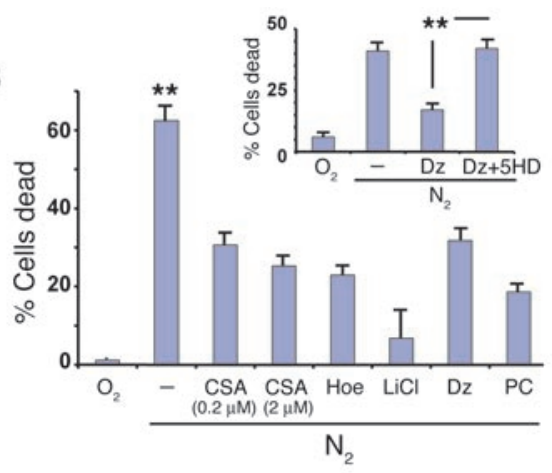

Membrane Soluble
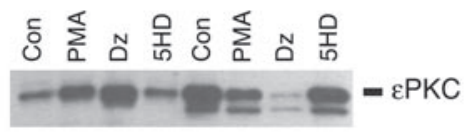

E
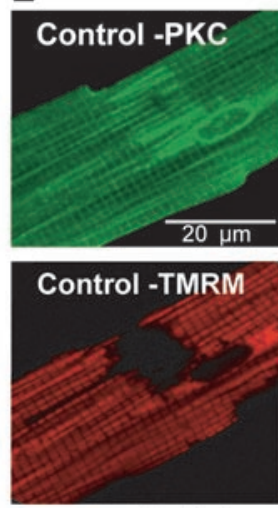

Control

linescan

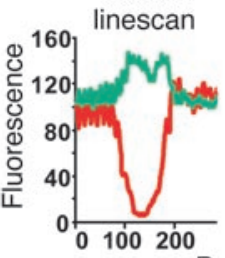

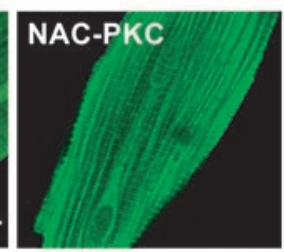

NAC-TMRM

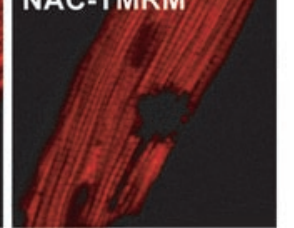

NAC

linescan

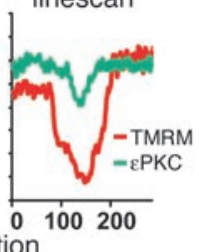

Figure 3

Mechanisms of protection. (A) MPT susceptibility to ROS $\left(t_{\mathrm{MPT}}\right)$ can be regulated by the

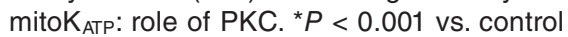
(Con). (B) Activation of distinct protection pathways improves cell survival to a similar degree in the hypoxia/reoxygenation protocol used to assess $t_{\text {MPT }}$. Hypoxia/reoxygenation groups included treatment with cyclosporin A, $\mathrm{Hoe}, \mathrm{Li}^{+}, \mathrm{Dz}$, and PC. The protective effect of $\mathrm{Dz}$ is abolished by $5 \mathrm{HD}$ (inset). ${ }^{* \star} P<0.02$. (C) Translocation of $\varepsilon P K C$ toward mitochondria, induced by mitoK $\mathrm{K}_{\text {ATP }}$ activation. The panels on the left represent immunostained cardiac myocytes $\left(15 \times 15 \mu \mathrm{m}^{2}\right.$ region surrounding the nucleus, shown for technical consistency). The immunoblot on the right shows that both Dz and PMA induce $\varepsilon$ PKC translocation from the soluble to the membranous cellular fraction. (D) Transmission electron microscopy of immunogold-labeled $\varepsilon$ PKC in a cardiac myocyte from a heart treated with PMA (100 $\mathrm{nM}, 15$ minutes), demonstrating mitochondrial membrane localization (right middle panel; dashed circle outlines a mitochondrial profile); immunolabeling is absent in control (not shown). (E) ROS-induced PKC translocation toward mitochondria. Photoexcitationmediated MPT induction in an approximately $10 \times 10 \mu \mathrm{m}^{2}$ region in TMRM-loaded cardiac myocytes (middle panels, red), and $\varepsilon \mathrm{PKC}$ immunostaining (top panels, green) in the same cells. The right panels show effects of the ROS scavenger NAC. The bottom panels compare the $\varepsilon$ PKC labeling through the photoexcited regions. within groups were made by an appropriate Student $t$ test, and $P$ less than 0.05 was taken to indicate statistical significance.

\section{Results}

Consequences of the ROS burst that occurs upon bypoxia/reoxygenation. Isolated cardiac myocytes remain viable without morphologic deterioration for at least 2 hours during exposure to $2-4 \% \mathrm{O}_{2}$. When isolated cardiac myocytes are loaded with TMRM and DCF and subjected to rapid reoxygenation after 1 hour of hypoxia, laser line-scanning confocal microscopy of a mitochondrial row shows that transmembrane potential $(\Delta \Psi)$ immediately increases for approximately 60 seconds (see also Supplement 2). After this brief phase, mitochondrial hyperpolarization abruptly stabilizes, at which point a significant increase in local ROS production is observed (the increase in DCF fluorescence; Figure 1A). After several minutes, a variable number of individual mitochondria develop a precipitous drop in $\Delta \Psi$ accompanied by an additional large ROS burst (a representative tracing from one mitochondrion is shown in Figure 1A), which we have previously demonstrated is caused by the MPT and the accompanying "ROS-induced ROS release" phenomenon (17). This demonstrates that reoxygenation after a period of prolonged hypoxia is sufficient to result in the pathologically increased endogenous production of ROS that culminates in MPT induction in a proportion of mitochondria.

Protection from bypoxia/reoxygenation injury by PC. Images of TMRM-loaded (and DCF-loaded) cardiac myocytes following reoxygenation after hypoxia for 1 hour (Figure 1B) show that the normally ordered arrays of active mitochondria (visualized by TMRM fluorescence in red) develop patterns punctuated by the scattered "holes" left by depolarized mitochondria after MPT induction; as can be seen in cells coloaded with DCF (in green), these holes correspond to sites of increased ROS. Remarkably, PC by exposure of cells to the mitoK $\mathrm{K}_{\mathrm{ATP}}$ opener $\mathrm{Dz}$ or to three 5 -minute cycles of hypoxia alternating with normoxia $60 \mathrm{~min}$ utes prior to the hour-long period of hypoxia largely prevented the mitochondrial depolarization. This protective effect of PC to 
A
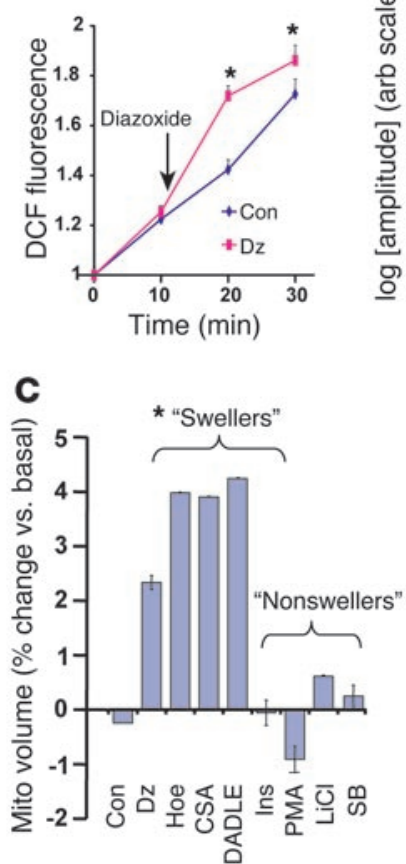

B

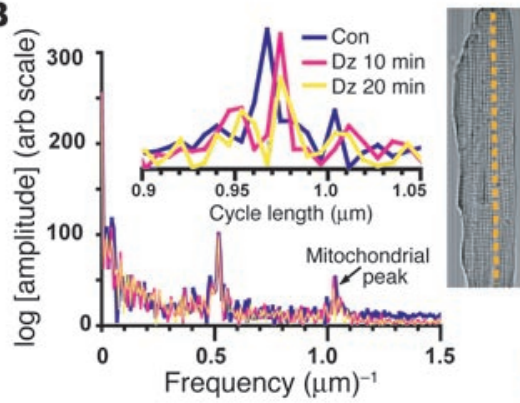

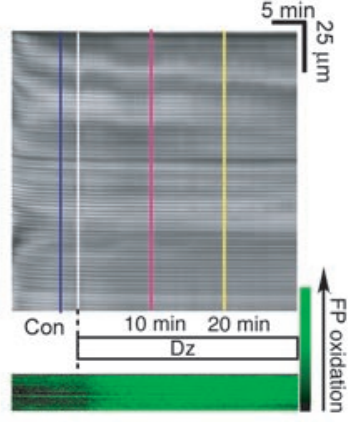

D
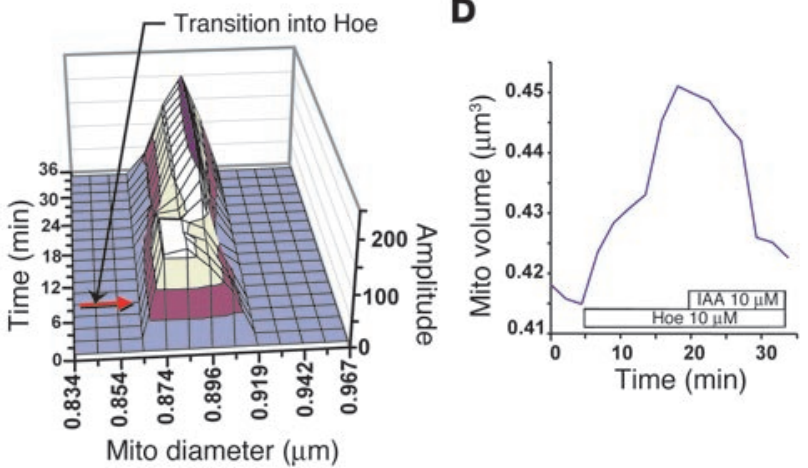

$\mathbf{E}$
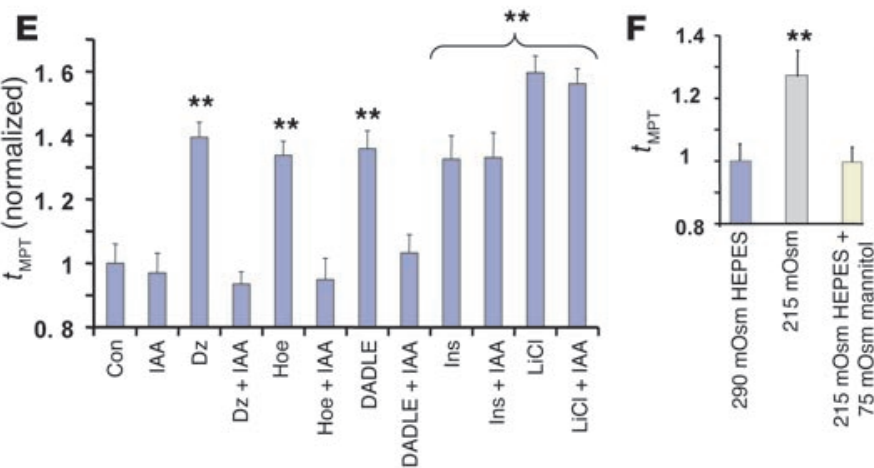

Figure 4

Mechanisms of protection dependent on mitochondrial swelling. (A) Enhanced ROS generation (in DCF-

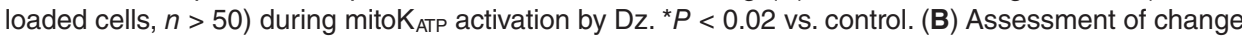
in mitochondrial volume after Dz treatment by Fourier analysis. Laser line-scan imaging of in situ mitochondria was performed along the long axis of the cell (right panels) during Dz exposure. High-resolution transmittance (gray image) and flavoprotein autofluorescence (488 $\mathrm{nm}$ excitation, green image) were obtained simultaneously. The left panel shows the Fourier frequency-domain spectrum from the transmittance line-scan data during the control period and periods of treatment with Dz for 10 and 20 minutes. The first-order peak indicates the regular sarcomere $Z$ structure. The spectrum inset enlarges the secondorder peak shifts (converted to micrometer scale), indicating small Dz-mediated changes in mitochondrial volume. Arb scale, arbitrary scale; FP flavoproteins. (C and D) Mitochondrial-volume changes induced by swellers and nonswellers ("SB" indicates SB 415286). C (right panel) and D show time-dependent volume changes after Hoe, and the reversal of the swelling effect by inhibition of $\mathrm{Cl}^{-}$transport using IAA94 (D). Ins, insulin. (E) Protection by mitochondrial swellers (but not by nonswellers) requires $\mathrm{Cl}^{-}$channel flux. (F) Osmotic change induces modulation of $t_{\mathrm{MPT}}$ measured under isotonic conditions and 15 minutes after transient (5 minutes) hypotonic conditions. ${ }^{* *} P<0.01$ (and all bars under brace) vs. control (E and $\mathbf{F}$ ).

MPT susceptibility to ROS governs cell survival. MPT induction in about one-third of cellular mitochondria by the local photoproduction of ROS (by laser photoexcitation of a precise area within a cell loaded with TMRM) causes approximately two-thirds of the population of cells exposed in this fashion to die within 1 hour (Figure 1C). Cell death results from MPT induction in a large proportion of cellular mitochondria regardless of whether it was caused by photoproduced ROS (Figure 1C) or by a ROS burst that occurred "naturally" (without photoexcitation) after hypoxia/reoxygenation (Figure 1, A and B; and see Figure 3B). The ROS scavenger Trolox or the NO donor S-nitroso- $N$ acetyl-penicillamine (SNAP) reduces the number of mitochondria that succumb to MPT induction (17, 27 ) to $18 \%$ or $21 \%$ and increases cell survival to $65 \%$ or $45 \%$, respectively. Significantly, Dz reduces this fraction of depolarized mitochondria to $22 \%$ and increases survival to $57 \%$, while 5 HD has an opposite effect, increasing the fraction to $41 \%$ and decreasing survival to $9 \%$ (Figure 1C). Thus, cardiac myocyte survival is steeply negatively correlated with the fraction of depolarized mitochondria due to MPT induction. It would be reasonable to hypothesize that mechanisms that control the ROS susceptibility to MPT induction, including those involving the mito $\mathrm{K}_{\mathrm{ATP}}$, could make a major impact on cell survival during ischemic stress.

The MPT ROS threshold is significantly reduced after bypoxia/reoxygenation. We have previously developed a means to quantify the susceptibility to the induction of the MPT by ROS in individual mitochondria inside cardiac myocytes (summarized in Figure 1D) (17). Repetitive laser scanning of a row of mitochondria in a cell loaded with TMRM causes additive, incremental exposure of just that laser-exposed area to the prevent MPT induction was fully abolished when the mitoK $\mathrm{K}_{\mathrm{ATP}}$ antagonist 5HD was present during exposure to $\mathrm{Dz}$ (not shown) or during the hypoxic PC protocol (Figure 1B). Thus, the protection enabled by both hypoxic PC and Dz prevents ROS induction of the MPT upon reoxygenation and shares the obligatory activation of the putative mitoK $\mathrm{K}_{\mathrm{ATP}}$. photodynamic production of ROS. After a reproducible ROS exposure, the MPT occurs, which is clearly identified by the immediate dissipation of $\Delta \Psi$ (Figure 1D) together with large-amplitude (pathological) mitochondrial swelling (as readily seen in isolated mitochondria; Figure 1E; ref. 28). It should be pointed out that the ROS-induced ROS release phenomenon is a consequence of MPT 


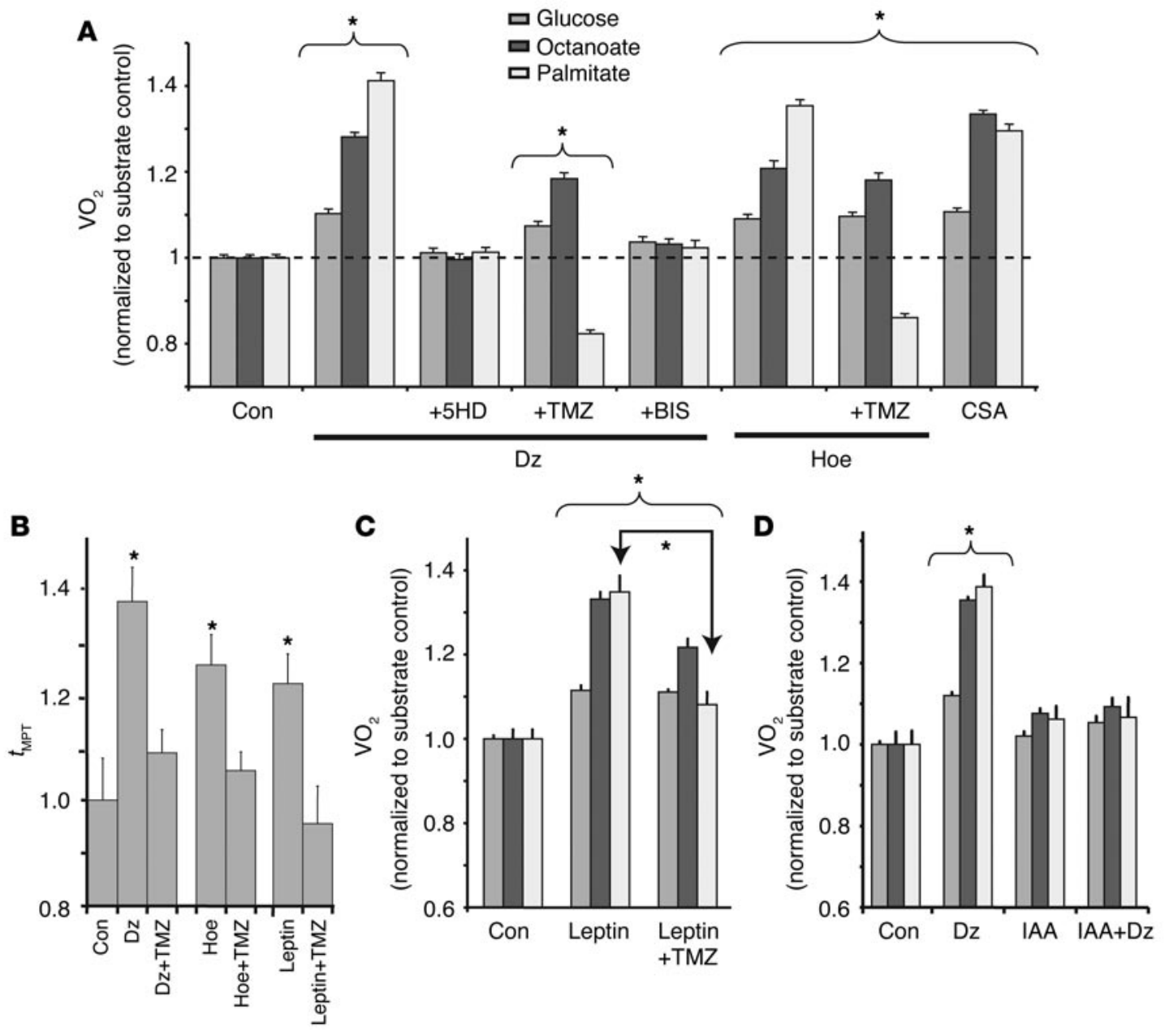

Figure 5

Relationship among mitochondrial swelling, metabolic flux/electron transport, and protection. (A) Mitochondrial swellers activate cell respiration. Three metabolic substrates were used: glucose, octanoate, and palmitate. Values indicated above the control bars are actual respiration rates (nmol $\mathrm{O}_{2} / \mathrm{min} / 10^{6}$ cells). (B) TMZ blocks protection by Dz, Hoe, and leptin in cardiac myocytes metabolizing palmitate. (C) Respiratory activation by leptin in cardiac myocytes metabolizing different substrates. (D) Sensitivity of Dz-induced respiratory activation to $\mathrm{Cl}^{-}$channel inhibition. ${ }^{\star} P<0.05$ (and all bars under brace) vs. control.

induction but is not necessary for the primary induction of the MPT by exogenous ROS (17), which is the phenomenon specifically being examined here using photoexcitation-generated ROS to trigger MPT induction. Thus, while Aon et al. (29) have elegantly shown that the propagation phase of ROS-induced ROS release could well require the inner membrane anion channel (IMAC), it is not involved in the current assessment of MPT ROS threshold, since the IMAC inhibitors 4,4'-diisothiocyanatostilbene-2,2'-disulfonic acid, disodium salt (DIDS) and PK11195 (29) do not affect MPT induction as performed here (data not shown).

In cardiac myocytes subjected to rapid reoxygenation after 1 hour of hypoxia $\left(2-4 \% \mathrm{O}_{2}\right)$, the MPT ROS threshold is significantly reduced to approximately $50 \%$ of the control levels immediately after restoration of normoxia, with a distinct trend toward further deterioration over the following 2 hours or longer (Figure 2, A and E). PC by transient exposure of cells to $\mathrm{Dz}(30 \mu \mathrm{M}$ for 15 minutes), or to the hypoxic PC protocol, largely prevented the reduction of MPT ROS threshold seen upon reoxygenation without PC (Figure $2, \mathrm{~B}, \mathrm{C}$, and $\mathrm{E})$. This protective effect of $\mathrm{PC}$ or its simulation with $\mathrm{Dz}$ to prevent MPT induction was fully abolished when the mitoK $\mathrm{KTP}_{\mathrm{AP}}$ antagonist 5HD or the ROS scavenger NAC was present during exposure to Dz or during the hypoxic PC protocol (Figure 2, D and E). Thus, the protection enabled by both hypoxic PC and Dz (a) prevents ROS induction of the MPT upon reoxygenation, (b) requires the formation of a ROS/redox signal to activate this protection, and (c) shares the obligatory activation of the putative mitoK $K_{\text {ATP. }}$

Enhancement of the MPT ROS threshold is the common link of protection. Not only does hypoxic PC or Dz prevent ROS induction of the MPT upon reoxygenation, but they each enhance the MPT ROS threshold over control by about $35-40 \%$ in the absence of the injury of hypoxia/reoxygenation (Figure 2E). For comparison purposes, the permeability transition pore (PTP) inhibitors cyclosporine A and SFA (13) (Figure 2F) and another mitoK $\mathrm{ATP}_{\text {agonist, }}$ pinacidil (Supplement 3), each produced the same range of protection. It should be noted that the protection by hypoxic PC and Dz (given transiently in advance of the pathological insult) exhibits a memory insofar as it extends up to several hours beyond completion of PC. Interestingly, while the protection of $\mathrm{Dz}$ and pinacidil 


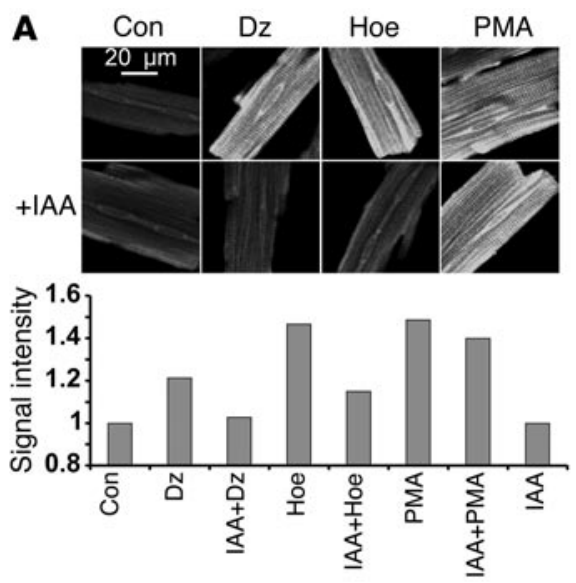

\section{B}

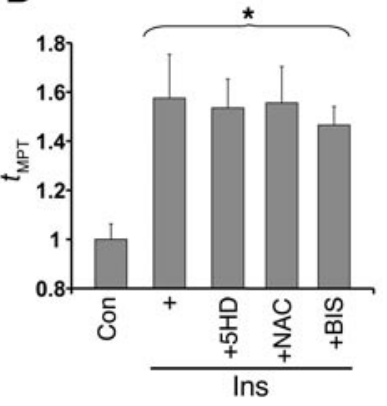

\section{Figure 6}

Distinct mechanisms of mitochondrial swelling-dependent and -independent protection. (A) The effect of $\mathrm{Cl}^{-}$channel inhibition on sweller-induced $\varepsilon$ PKC translocation. Translocation by the nonsweller PMA is insensitive to IAA94. (B) Mitochondrial swelling-independent protection does not require the mitoK $\mathrm{K}_{\mathrm{ATP}} \mathrm{ROS}$, or PKC. (C and D) Reversal of insulin-induced and $A_{1}$ agonistinduced (CCPA-induced) protection by inhibitors of PI3K (wortmannin [wort] and LY 294002) and mTOR (rapamycin [rap]). CCPA protection is also mediated in parallel by swelling-independent PKC and mitoK $\mathrm{K}_{\text {ATP }}$ pathways. (E) GLP-1-induced protection is mediated by PKA and blocked by the PKA inhibitor Rp-8-CPT-cAMPS (Rp). ${ }^{\star} P<0.01$ (and all bars under brace) vs. control.
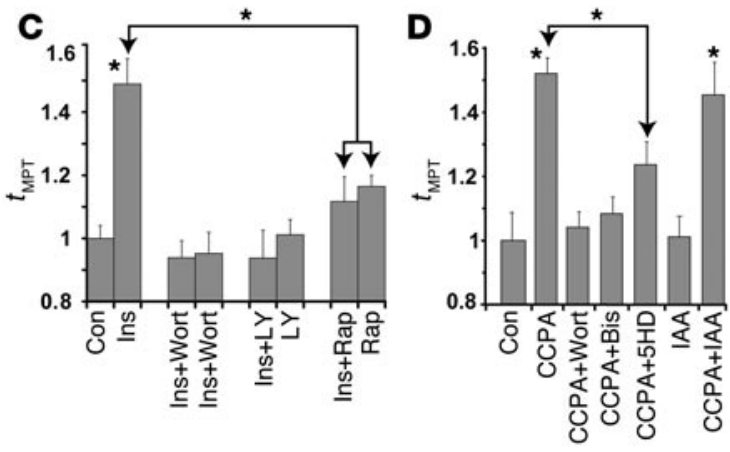

$\mathbf{E}$

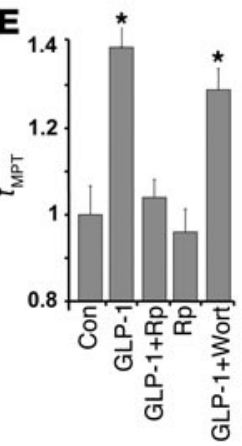

achieved 60-65\%, whereas hypoxic PC, Dz, Hoe, cyclosporin $\mathrm{A}$, and $\mathrm{Li}^{+}$reduced this by at least half (Figure 3B). Notably, 5HD blocked the protection afforded by $\mathrm{Dz}$ (Figure 3B, inset).

Protection is triggered by pathways dependent on and independent of mitochondrial swelling. Whereas the protective mechanisms of hypoxic PC, Dz, pinacidil, Hoe, DADLE, cyclosporin A, SFA, and bradykinin to enhance the MPT ROS threshold exhibit a memory of up to several hours (i.e., each works as PC), those of insulin, IGF-1, adenosine $\mathrm{A}_{1}$ agonist (CCPA), and GLP-1 do not have a significant memory (Supplement 4). This suggested

can be inhibited by 5HD, it is also inhibited by the PKC inhibitor bisindolylmaleimide I (BIS), and by NAC (Figures 2E and 3A; Supplement 3). Furthermore, this protective effect of $\mathrm{Dz}$ is mimicked by PKC activation via PMA exposure, which can be inhibited by $5 \mathrm{HD}$ (Figure $3 \mathrm{~A}$ ). These results suggest that $\mathrm{PKC}$ activation is potentially both upstream and downstream of mitoK $\mathrm{K}_{\mathrm{ATP}}$ mechanisms of protection. Hypothesizing that enhancement of the MPT ROS threshold is the common mechanism of protection, we tested a broad range of pharmacologic treatments that have been demonstrated to reduce the size of experimental infarction of the heart or brain. We found that diverse cardio/neuroprotective agents such as $\mathrm{Na} / \mathrm{H}$ exchange (NHE) inhibitors (Hoe694 and Hoe642), cyclosporin A, SFA, receptor tyrosine kinase ligands (insulin and IGF-1), $G$ protein-coupled receptor ligands (e.g., CCPA $\left[\mathrm{A}_{1}\right]$, bradykinin $\left[\mathrm{B}_{2}\right], \delta$-opioid, DADLE, and GLP-1), and inhibitors of GSK-3 $\beta$ ( $\mathrm{Li}^{+}, \mathrm{SB} 216763$, and SB 415286), each likely signaling by distinct upstream pathways, all enhance the MPT ROS threshold over control by about $35-50 \%$ (see below), suggesting that this is the common link in protection against ischemic injury.

The ability to enhance MPT ROS threshold predicts a positive effect on cell survival after bypoxia/reoxygenation. Since (a) hypoxia/reoxygenation causes a ROS burst that induces many mitochondria to undergo the MPT and that significantly reduces the MPT ROS threshold of the remaining functional mitochondria, (b) MPT induction in a significant fraction of cellular mitochondria results in cell death, and (c) both hypoxic PC and pharmacologic PC were able to prevent the reduction in MPT ROS threshold, we examined the effect of hypoxic PC and those pharmacologic treatments that enhance the MPT ROS threshold on the survival of cardiac myocytes following hypoxia/reoxygenation. After 3 hours of reoxygenation following 1 hour of hypoxia $\left(2-4 \% \mathrm{O}_{2}\right)$, the fraction of dead myocytes two general upstream signaling mechanisms of protection that converge on the permeability transition pore complex.

Because the MPT protection of hypoxic PC and Dz (or pinacidil; Supplement 3 ) is dependent on ROS and PKC signaling (Figures $2 \mathrm{E}$ and $3 \mathrm{~A}$ ), we examined whether these mechanisms are shared by the group exhibiting PC memory. Immunolabeling experiments show that $\mathrm{Dz}$ causes the translocation of the $\mathrm{PPKC}$ (which has been implicated to mediate PC; ref. 30), comparable to that seen with PMA (Figure 3C), particularly to the mitochondria (Figure 3D). Similar patterns of PKC translocation are also seen with hypoxic PC, Hoe, DADLE, and cyclosporin A (not shown). Furthermore, as with hypoxic PC and Dz, the memory-associated MPT protection exerted by Hoe, DADLE, and cyclosporin A was blocked by NAC (not shown), strengthening the case for the possibility of common redox signaling mechanisms effected by this group. Since PKC and other signaling molecules are recognized to be redox regulated, we examined whether local ROS production in cardiac myocytes could induce PKC translocation. Photoexcitation of a discrete region of about $10 \times 10 \mu \mathrm{m}^{2}$ in TMRM-loaded myocytes beyond the point of MPT induction was found to be sufficient to induce the translocation of $\varepsilon$ PKC inside that same region (Figure $3 \mathrm{E}$ ). In contrast, photoexcitation (sufficient to induce the MPT) in the presence of NAC was not associated with PKC translocation (Figure 3E), suggesting that local ROS/redox signaling, capable of being buffered by NAC, can mediate PC.

Given the apparent role played by ROS in PC, we measured ROS production in cardiac myocytes loaded with DCF during the PC induction phase. Figure 4A shows the cellular production of ROS induced by the prototypical PC-inducing agent, Dz, in agreement with previous observations (31). Since mitochondria are one of the major cellular sources of ROS (32), and Dz activates the putative 


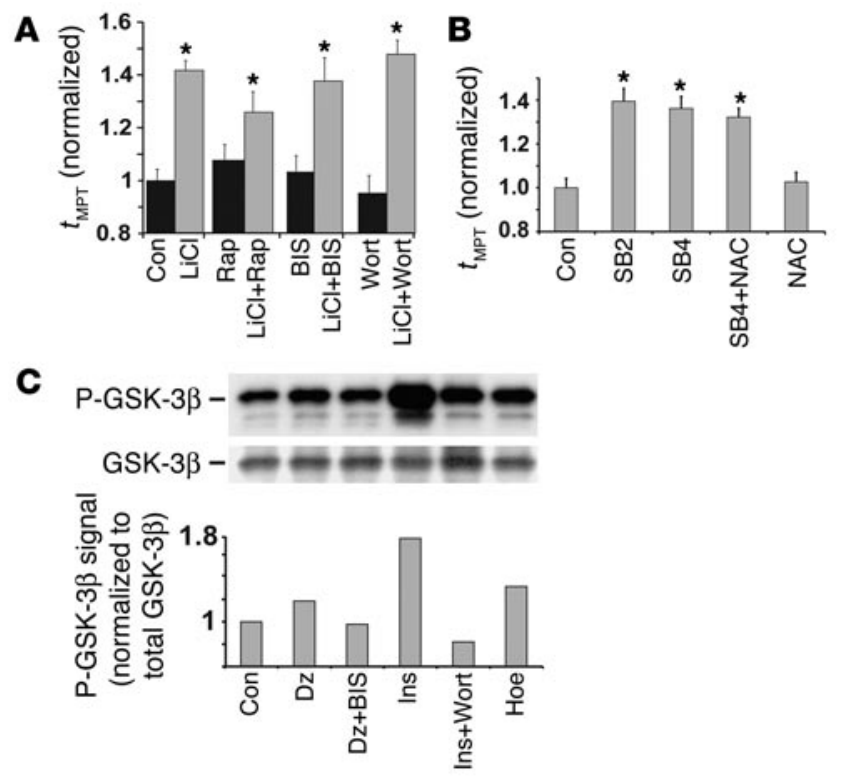

Figure 7

Central role of GSK-3 $\beta$ in protection. (A and $\mathbf{B})$ Protection induced by $\mathrm{Li}^{+}$, SB 216763 (SB2), or SB 415286 (SB4) cannot be reversed by $\mathrm{PKC}, \mathrm{PI3K}$, or mTOR inhibitors $(\mathbf{A})$ or by NAC (B), respectively. ${ }^{*} P<0.05$ vs. control. (C) Phosphorylation of GSK-3 $\beta$ (serine-9) in cell lysates, caused by pharmacologic PC. The immunoblot data are representative of three independent experiments.

mito $_{\text {ATP }}$ that has been shown to cause swelling in isolated mitochondria (33), we developed a novel single-cardiac myocyte imaging technique that proves that exposure to certain PC agents causes acute regulatory mitochondrial swelling (by up to 4\%) in situ (Figure 4B), and these effects on mitochondrial volume are summarized in Figure 4C (see Supplement 1 for further details). Remarkably, this analysis indicates that PC with a memory - simulated by Dz, Hoe, DADLE, and cyclosporin A (and by pinacidil and bradykinin, not shown), a group we will call "swellers" - is associated with an approximately $2.5-4 \%$ increase in mitochondrial volume, whereas protection without a durable memory - simulated by insulin, $\mathrm{PMA}, \mathrm{Li}^{+}$, and the specific, small-molecule GSK-3 inhibitors SB 216763 and SB 415286 (and by IGF-1, not shown) - is not associated with increased mitochondrial volume (Figure 4C). Furthermore, the kinetics of swelling (shown in Figure 4, C and D, for Hoe as the example representative of swellers) is rapid, occurring within minutes of exposure.

This raises the realistic possibility that the mitochondrial swellers Hoe, DADLE, and bradykinin act directly on mitochondrial targets, just as Dz, pinacidil, cyclosporin A, and SFA do. In the present model with the resting cardiac myocytes, it seems unlikely that the site of Hoe action that initiates MPT protection would be the plasma membrane NHE1, particularly since we have previously demonstrated that intracellular $\mathrm{pH}$ (and consequently $\mathrm{Ca}^{2+}$ ) remains unchanged after Hoe exposure in these cells (34). Furthermore, assuming that $\mathrm{Dz}$ and pinacidil increase mitochondrial matrix $\mathrm{K}^{+}$ (via activation of the mito $\mathrm{K}_{\mathrm{ATP}}$ ), and that Hoe could effectively act in the same manner by inhibiting net mitochondrial $\mathrm{K} / \mathrm{H}$ exchange, then the maintenance of electroneutrality would obligate the influx of a counterion, most likely $\mathrm{Cl}^{-}$. This accumulating excess in matrix solute (largely $\mathrm{KCl}$ ) would in turn cause the osmotic influx of water, resulting in the observed mitochondrial swelling.
The next series of experiments was performed with the selective $\mathrm{Cl}^{-}$channel blocker IAA94 to examine the mechanism of the mitochondrial swellers, and the role of swelling in memory-associated MPT protection. IAA94 was able to reverse or prevent the increase in mitochondrial volume induced by swellers (Figure 4D shows Hoe as a representative example). Importantly, IAA94 completely prevented MPT protection induced by the swellers Dz, Hoe, and DADLE but had no effect on the MPT protection afforded by the nonswellers insulin and $\mathrm{Li}^{+}$(Figure 4E). That MPT protection can be induced by transient exposure of cardiac myocytes to hypotonic buffer solutions (but not by diluted electrolyte solutions with physiologic osmolarity restored by mannitol) provides further evidence that mitochondrial swelling is an essential part of the mechanism of memory-associated PC (Figure 4F).

Mostly based on the results of experiments on isolated mitochondrial suspension, it was suggested that $\mathrm{Ca}^{2+}$ is an important factor in MPT induction $(11,35)$. However, in cardiac myocytes with an intact sarcolemma, we found that $\mathrm{Ca}^{2+}$ concentrations over a very wide range do not appreciably affect the MPT ROS threshold (see Supplement 5).

It has been argued that mitochondrial swelling could enhance electron transport (36) by activation of metabolic flux (i.e., of glucose and fatty acids). Since the mitochondrial swellers maintain $\Delta \Psi$ in intact adult cardiac myocytes without depolarization (or even cause a small increase in $\Delta \Psi$ ), this activation would be accompanied by an obligatory increase in mitochondrial ROS production, which could provide the redox signal responsible for PKC activation and MPT protection. In cardiac myocyte suspension, we found that swellers (Dz, Hoe, and cyclosporin A) increased oxygen consumption $\left(\mathrm{VO}_{2}\right)$ over base line by about $10 \%, 25-30 \%$, and about $35 \%$ when utilizing glucose, the medium-chain fatty acid octanoate, or the long-chain fatty acid palmitate, respectively (Figure 5A). Furthermore, the $\mathrm{VO}_{2}$ increases following $\mathrm{Dz}$ (Figure $5 \mathrm{~A}$ ) and DADLE (not shown) were prevented by blocking of the mito $_{\text {ATP }}$ or by inhibition of PKC. The partial fatty acid oxidation (PFAO) inhibitor TMZ prevented both the sweller-induced $\mathrm{VO}_{2}$ increase (Figure 5A) and MPT protection in palmitate, but not in glucose or octanoate (Figure 5B, shown only for palmitate). Significantly, the nonswellers (i.e., insulin and $\mathrm{Li}^{+}$; not shown) made a minimal impact on $\mathrm{VO}_{2}$.

Leptin was recently shown to stimulate fatty acid oxidation and uptake of glucose $(37,38)$. In the present model, leptin fully simulated the pattern of sweller-induced $\mathrm{VO}_{2}$ increase (Figure 5C) and MPT protection (Figure $5 \mathrm{~B}$ ) with the responses in palmitate being largely blocked by TMZ. This confirmed the concept that, whether the increased oxidation of fatty acids and uptake of glucose are driven by leptin or by swelling, the enhancement of electron transport is sufficient to induce memory-associated MPT protection and thus could be an integral part of the swelling-induced mechanism. That $\mathrm{Cl}^{-}$channel inhibition was able to prevent mitochondrial swelling (Figure 4D), MPT protection (Figure 4E), the increase in $\mathrm{VO}_{2}$ (Figure $5 \mathrm{D}$, with $\mathrm{Dz}$ as an example), and the PKC translocation (Figure 6A) induced by the sweller group supports the concept that moderate, reversible mitochondrial swelling per se causes increased respiration and consequent redox activation of $\mathrm{PKC}$, which results in memory-associated MPT protection.

Mechanisms of mitochondrial swelling-independent protection are distinct from mechanisms controlled by mitochondrial swelling. As discussed above, insulin, IGF-1, CCPA, GLP-1, PMA, Li ${ }^{+}$, and SB (216763 and 415286) exert protection without causing mitochondrial swelling 

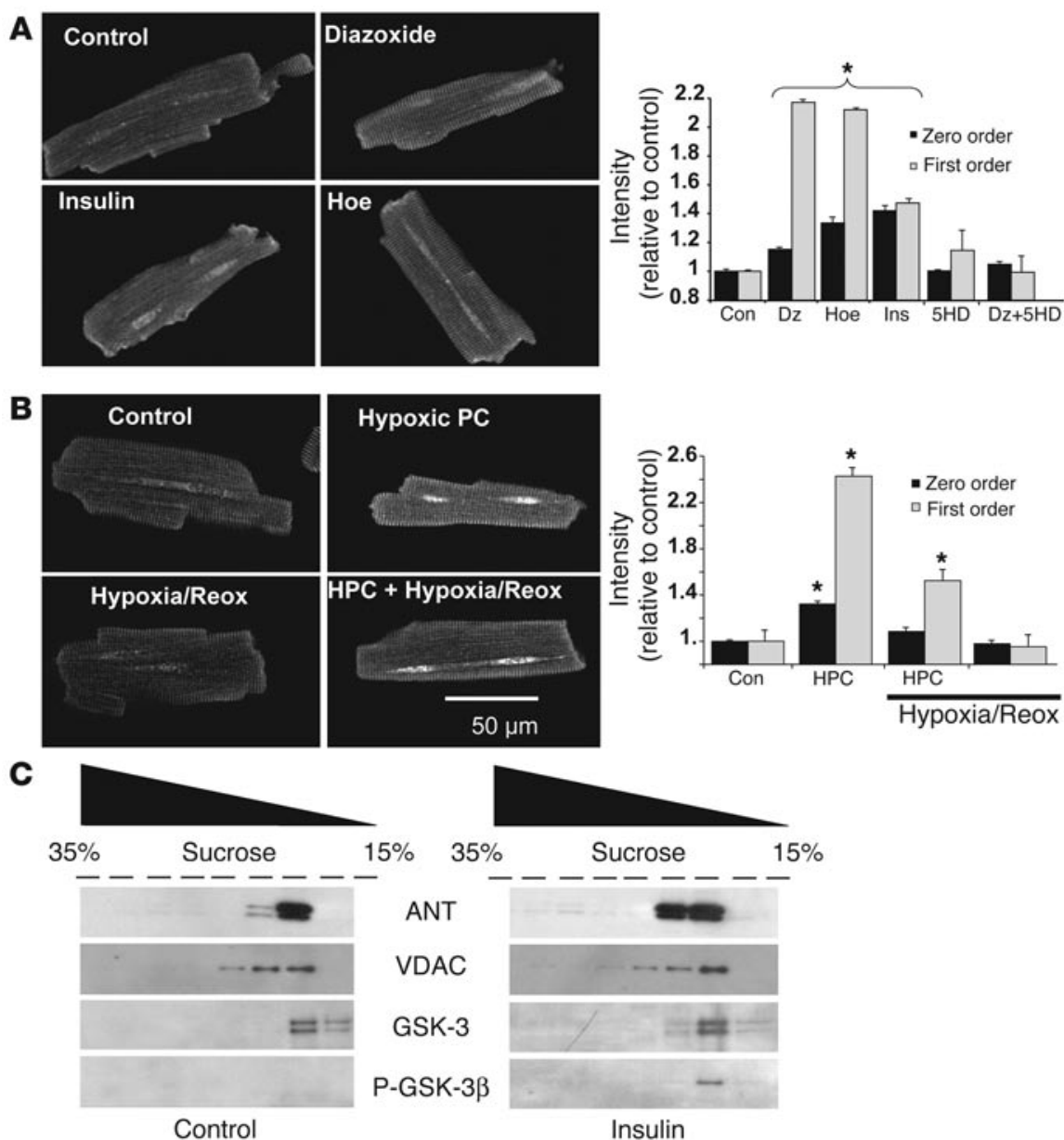

ANT

VDAC

GSK-3

P-GSK-3B

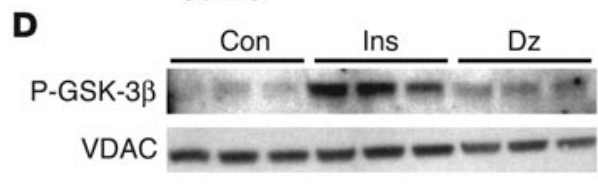

$15 \%$

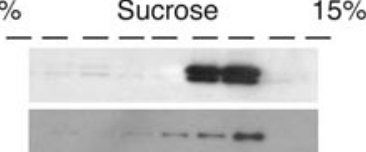

\section{Figure 8}

Localization and regulation of a mitochondrial GSK-3 $\beta$ pool during protection signaling. (A and B) P-GSK-3 $\beta$ immunocytochemical labeling: changes in average versus compartmentalized signal intensity determined from 2D Fourier analysis (see text; images of cells exposed to 5HD and to Dz plus 5HD not shown). ${ }^{\star} P<0.01$ (all bars under brace) vs. respective control. (C) Immunoblots of mitochondrial membrane sucrose gradient fractions, from control and insulin-treated $(30 \mathrm{nM})$ rat hearts, probed with adenine nucleotide translocator (ANT), voltage dependent anion channel (VDAC), GSK-3, and P-GSK-3 $\beta$. (D) Immunoblot of total mitochondrial proteins isolated from control, insulin-treated (30 nM), and Dz-treated ( $30 \mu \mathrm{M})$ rat hearts. ${ }^{* *} P<0.03$ vs. control.

tection without mitochondrial swelling that cannot be prevented by NAC and by inhibitors of PKC, PI3K, or mTOR/p70 ${ }^{\mathrm{s} 6 \mathrm{~K}}$ (Figure $7, A$ and $B$ ) and thus appear to be acting on a target downstream of all the other protective agents and pathways examined, we hypothesized that GSK-3 could be this point of integration. Since it was recently shown that GSK-3 $\beta$ resides inside mitochondria from nonmyocyte cell lines (41), we examined the role of this isoform in protection signaling.

Convergence of distinct protection mechanisms on GSK-3 $\beta$. The PC mitochondrial swellers (represented by Dz and Hoe), and the protective nonswellers (represented by insulin), are each capable of phosphorylating GSK-3 $\beta$ on regulatory serine-9 (Figure 7C). Furthermore, the Dz- and insulin-mediated increases in P-GSK-3 $\beta$ signal are abolished by inhibition of PKC and PI3K, respectively. The increases in whole-cell P-GSK-3 $\beta$ sig-

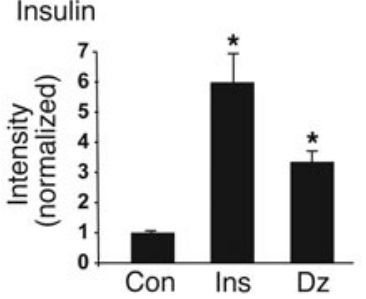

or a durable memory. In addition, the protection they afford is not affected by inhibition of the mito $\mathrm{K}_{\mathrm{ATP}}$, the "swelling-permissive" $\mathrm{Cl}^{-}$channel, ROS, or PKC (except for PMA, which activates PKC and requires some functional participation of the mitoK $_{\mathrm{ATP}}$; see Discussion) (Figure 4E, and Figure 6B, showing insulin as an example). Interestingly, receptor tyrosine kinase activation via insulin (or IGF-1) or $G$ protein-coupled receptor activation via CCPA $\left(\mathrm{A}_{1}\right)$ elicits protection via $\mathrm{PI} 3 \mathrm{~K} / \mathrm{PKB} / \mathrm{Akt}$ and $\mathrm{mTOR} / \mathrm{p} 70^{\mathrm{s} 6 \mathrm{~K}}$ (Figure 6, $C$ and $D)$, whereas $G$ protein-coupled receptor activation via GLP-1 acts through PKA-dependent pathways (Figure 6E). $A_{1}$ signaling is also mediated in parallel by PKC and mitoK $_{\text {ATP }}$ mechanisms but not mitochondrial swelling (Figure 6D). This diversity of upstream signaling pathways (i.e., PKA, PKB, PKC), which must all converge to cause a similar degree of functional protection of the permeability transition pore complex (the end effector), suggested that a point of integration on a master switch immediately proximal to the permeability transition pore complex could be involved. Because $\mathrm{Li}^{+}$(a selective GSK-3 inhibitor at the dose used; ref. 39) (Figure 7A) and SB (216763 and 415286) (40) (Figure 7B) can exert potent MPT pro- nals are modest but reproducibly observed (20-30\% over base line) with $\mathrm{Dz}$ and Hoe. Because the cell lysate immunoblot would but poorly resolve critical changes in local signaling in a small compartment of an otherwise large pool of a multifunctional switching nexus such as GSK-3 $\beta$, we performed immunostaining of isolated cardiac myocytes. Immunocytochemical labeling shows a dramatic increase in a distinct sarcomeric/mitochondrial pattern of P-GSK-3 $\beta$ signals after exposure to Dz and Hoe (Figure $8 \mathrm{~A}$ ) and to hypoxic PC (Figure 8B). Quantification of this compartmentalized signal by two-dimensional Fourier analysis reveals that the P-GSK-3 $\beta$ signals increase more than twofold (vs. control) in a subcellular pattern compatible with a mitochondrial distribution in response to Dz, Hoe, and hypoxic PC (Figure 8, A and B). Notably, this P-GSK-3 $\beta$ signal to Dz was blocked by 5HD (Figure $8 \mathrm{~A}$ ). While this signal after PC persists above control even after 1 hour of hypoxia/reoxygenation, it is notable that hypoxia/reoxygenation alone does not increase P-GSK-3 $\beta$ (Figure 8B).

GSK-3 is highly active in unstimulated cells and becomes inactivated in response to mitogenic stimulation (reviewed in ref. 22). 
A
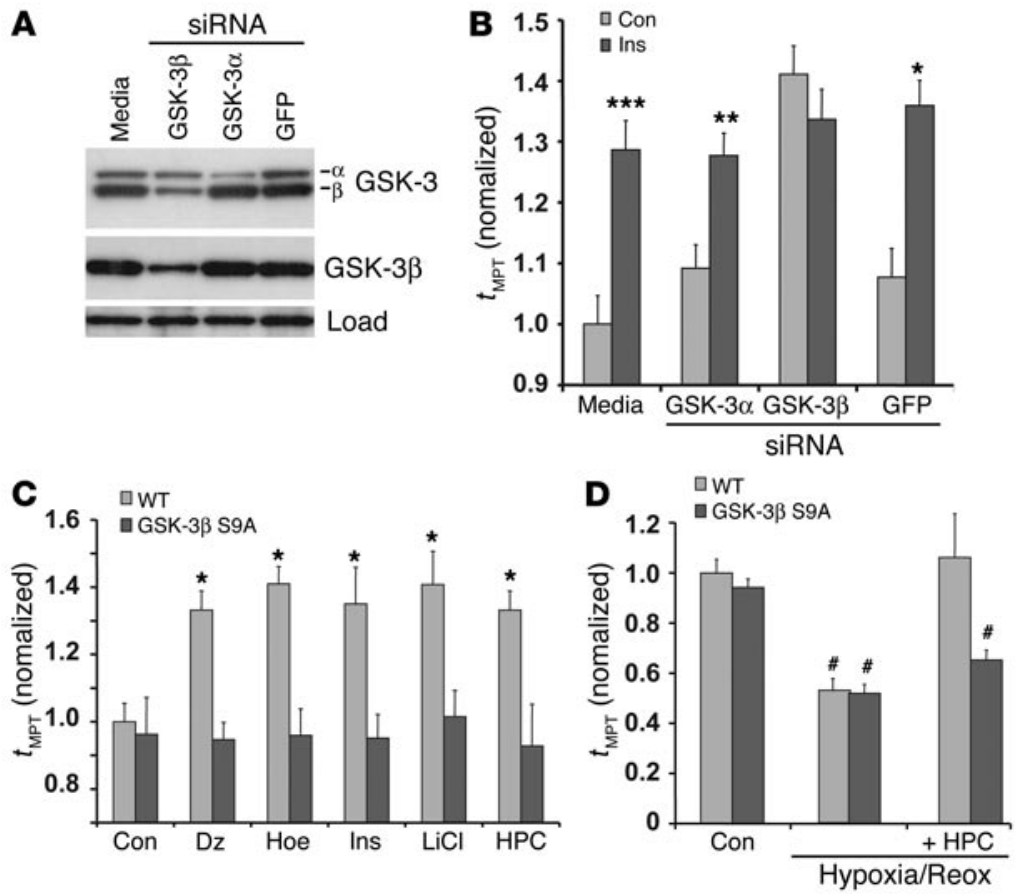

Figure 9

GSK-3 $\beta$, and not GSK-3 $\alpha$, regulates the protection state. (A) SiRNA treatment specifically decreases respective protein levels of GSK- $3 \alpha$ and GSK- $3 \beta$ in neonatal rat cardiac myocytes; GFP siRNA and media alone served as negative controls. Immunoblot of cell lysates probed with antibodies against GSK-3 $\alpha / \beta$, GSK-3 $\beta$, and $39-\mathrm{kDa}$ subunit of mitochondrial Complex I (as a loading control). The immunoblot is representative of two independent experiments. (B) Silencing of GSK-3 $\beta$, but not of GSK-3 $\alpha$, enhances $t_{\mathrm{MPT}}$ to levels comparable to insulin-induced protection in neonatal rat cardiac myocytes. Data comprise two independent experiments; $n=30$ in each group (except $n=22$ for GFP siRNA). ${ }^{*} P<0.02,{ }^{* \star} P<0.001$, ${ }^{\star * \star} P<0.0001$ vs. respective control. (C) Constitutive activation of GSK-3 $\beta$ prevents ability to engage protective signaling. GSK-3 $\beta$ inhibition is required for protection against oxidative stress. Both mitochondrial sweller-dependent and-independent protection mechanisms are abolished in adult cardiac myocytes from GSK-3 $\beta$ S9A TG mice. ${ }^{\star} P<0.02$ vs. control. (D) Hypoxic PC protection after hypoxia/reoxygenation is abolished in adult cardiac myocytes from GSK-3 $\beta$ S9A TG mice. $P<0.01$ vs. control.
Inactivation of GSK-3 by phosphorylation usually results in activation of downstream signaling pathways. The activity of GSK-3 $\beta$ is inversely related to the phosphorylation status of serine-9 (42). Dephosphorylation of this site, or mutations that prevent phosphorylation, result in activation of the kinase. Because the MPT protection data indicated that GSK-3 $\beta$ acts on a mitochondrial target, we examined whether GSK-3 $\beta$ is localized on cardiac mitochondria. Using sucrose gradient fractionation of isolated cardiac mitochondrial membranes followed by immunoblotting, we found (a) that GSK-3 $\beta$ is localized on mitochondria, and possibly associated with components of the permeability transition pore complex (specifically, adenine nucleotide translocator and voltage-dependent anion channel [Figure 8C], as well as hexokinase and creatine kinase [not shown]) given that they cosegregate in the same fraction, and (b) that this mitochondrial GSK-3 $\beta$ pool becomes serine-9-phosphorylated after the heart is exposed to $\mathrm{Dz}$ and insulin (representing the mitochondrial swelling and nonswelling modes of protection signaling, respectively; Figure 8, C and D).

Both GSK-3 isoforms, GSK-3 $\alpha$ and GSK-3 $\beta$, are expressed in rat cardiac myocytes (Figure 9A), and they may be similarly regulated and may have very similar targets (21). Because none of the tested drugs that exert MPT protection, nor the direct GSK-3 inhibitors $\mathrm{Li}^{+}$and SB (216763 and 415286), are isoform-specific inhibitors, we used RNA silencing separately for both GSK-3 isoforms to verify that GSK- $3 \beta$ and not GSK- $3 \alpha$ is involved in the regulation of the cell-protection state. Just as Dz, Hoe, insulin, and many other drugs inactivate GSK-3 $\beta$ by phosphorylation, which results in the apparent activation of downstream cell-protection pathways, we expected that knocking down the levels of enzyme should be the functional equivalent and should similarly reveal the protection state. Specific siRNAs targeted separately against each GSK-3 $\alpha$ and GSK-3 $\beta$ were used to independently reduce protein levels of individual GSK-3 isoforms in neonatal cardiac myocytes. SiRNA against GFP (a protein not expressed in these cells) was used as a negative control for the RNA silencing technique. Insulin was used in each group as a competent positive control to induce the full protection state. The data show that knock-down of GSK-3 $\alpha$ (by $\sim 75 \%$ ) does not in itself produce the protection state, since insulin still achieved protection in these cells, whereas knock-down of GSK-3 $\beta$ (by $\sim 75 \%$ ) by itself achieves levels of protection comparable to that seen with insulin; in fact, insulin cannot further increase the level of protection above that already achieved in these cells. There is no significant effect of the RNA interference technique on protection signaling as revealed by the GFP controls. Thus, the reduction in the activity of the $\beta$ isoform of GSK3 (through changes in either specific activity or level of enzyme), but not of the $\alpha$ isoform, mediates MPT protection signaling in the present model (Figure 9, A and B).

To further prove that GSK-3 $\beta$ serves as the integration point of the distinct signaling pathways that mediate protection in the adult heart, we used TG mice with cardiac-specific expression of a constitutively active (signal-resistant) form of GSK-3 $\beta$, containing a serine-9-to-alanine mutation (24). While hypoxic PC, Dz, Hoe, insulin, and $\mathrm{Li}^{+}$were each capable of promoting MPT protection by $35-40 \%$ in cardiac myocytes from WT mice, this benefit was completely absent in TG mouse myocytes (Figure 9C). Hypoxia/ reoxygenation injury reduced the MPT ROS susceptibility to approximately $50 \%$ of the control levels immediately after restoration of normoxia in cells from both WT and TG mice, a reduction comparable to that seen using cardiac myocytes from rats (Figure $2 \mathrm{E})$. However, while hypoxic PC effectively prevented the reduction of MPT ROS threshold seen upon reoxygenation in WT cells, this benefit was completely absent in TG mouse myocytes (Figure 9D). Thus, hypoxic PC, as well as a broad range of pharmacologic protection mechanisms against hypoxia/reoxygenation injury and oxidant stress, requires functionally inhibitable GSK-3 $\beta$.

\section{Discussion}

Cell protection involves activation of endogenous signaling, which can confer significant resistance to oxidant and other stresses asso- 


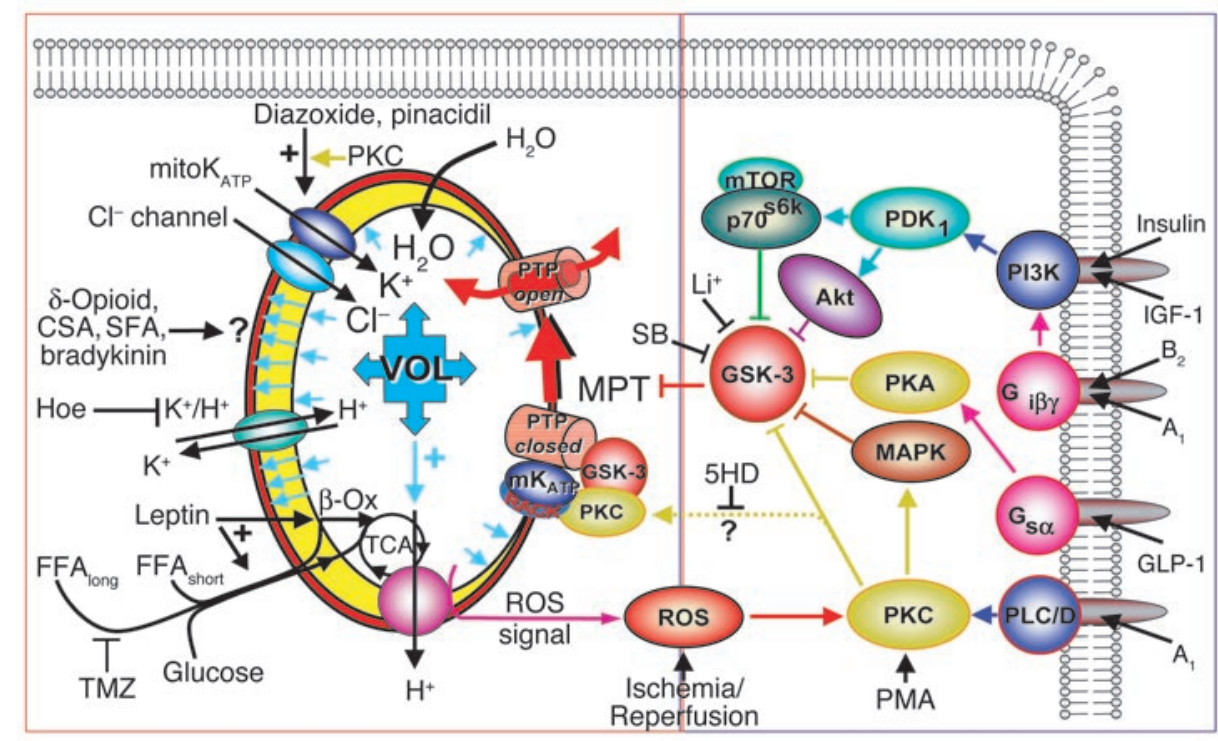

\section{Figure 10}

The integrated pathways of protection. Schematic showing the principal mechanisms: pathways dependent on change in mitochondrial volume ("swellers" such as Dz, pinacidil, leptin, DADLE, Hoe, and cyclosporin A; left, outlined in red) and pathways independent of change in mitochondrial volume ("nonswellers" such as PMA, insulin, IGF-1, CCPA, GLP-1, Li+, SB 216763, and SB 415286; right, outlined in blue). The convergence of these pathways via inhibition of GSK- $3 \beta$ on the end effector, the permeability transition pore (PTP) complex, to limit MPT induction, is the general mechanism of protection. VOL, volume; $\beta$-Ox, $\beta$-oxidation; TCA, tricarboxylic acid cycle; PLC/D phospholipase C and phospholipase D. ciated with hypoxia/reoxygenation,thus promoting the enhanced capacity for cell survival. However, the upstream signaling mechanisms have remained an area of active debate, and the end effector(s) has remained unidentified. Here we show that hypoxia/reoxygenation significantly reduces the ROS threshold for MPT, that cardiac myocyte survival is steeply negatively correlated with the fraction of depolarized mitochondria, and that a wide variety of cardio/neuroprotective agents acting via distinct upstream mechanisms all promote cell survival by limiting MPT induction. We demonstrate that protection can be triggered in two general ways - dependent on and independent of regulatory mitochondrial swelling - which converge via inhibition of GSK-3 $\beta$ on the end effector, the permeability transition pore complex, preventing the MPT (Figure 10).

Protection exhibiting a memory (i.e., PC) can be induced by a wide spectrum of triggers. We have defined protection with a durable memory as follows: the ability of an agent to demonstrate an increase in MPT ROS threshold (i.e., protection) at least an hour beyond the time when the agent is washed from the system. This is compatible with the concept of preconditioning. Protection without a durable memory is characterized by agents that can increase MPT ROS threshold during the actual period of exposure, but this protection returns to base-line levels with minutes (formally, $<15$ minutes) after washout. The latter agents would act poorly in terms of the ability to precondition. Within such a definition, durable memory can be induced by transient hypoxia. It can also

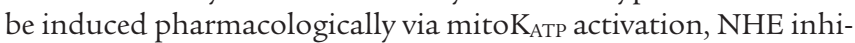
bition (which may work directly on mitochondria via inhibition of $\mathrm{K} / \mathrm{H}$ exchange), activation of bradykinin $\mathrm{B}_{2}$ or $\delta$-opioid receptors, as well as cyclosporin A and SFA. All of these induce memory, in turn, via mitochondrial $\mathrm{Cl}^{-}$flux regulated swelling causing enhanced substrate oxidation (palmitate $\geq$ octanoate > glucose), mitochondrial electron transport and ROS production, leading to redox activation of PKC, which in turn inhibits GSK-3 $\beta$ ). At the same time, memory can be induced "biotechnologically" (by reducing GSK-3 $\beta$ activity through RNA interference, or by S9A substitution in the GSK-3 $\beta$ molecule). Alternatively, protection that does not exhibit a long-lasting memory (and occurs independently of upstream signaling by mitochondria) is elicited by receptor tyrosine kinase activation via insulin and IGF-1, or by G protein-coupled receptor activation via adenosine $A_{1}$ receptors, signaling through $\mathrm{PI} 3 \mathrm{~K} / \mathrm{PKB} / \mathrm{Akt}$ and $\mathrm{mTOR} / \mathrm{p} 70^{\mathrm{s} 6 \mathrm{~K}}$ (with $\mathrm{A}_{1}$ signaling in parallel via $\mathrm{PKC}$ ), whereas $\mathrm{G}$ protein-coupled receptor activation via GLP-1 acts through PKA-dependent pathways. PMA, $\mathrm{Li}^{+}$, and SB (216763 and 415286) act directly on their downstream effectors (PKC and GSK-3) and hence exert protection without a memory (Figure 10). These findings with CCPA in rat cardiac myocytes are in accordance with published data (43-45) (and in each case, CCPA does not reproduce the ability of adenosine to precondition in the rat or the rabbit).

Diverse upstream signaling pathways (i.e., feeding into PKA, $\mathrm{PKB}$, or $\mathrm{PKC}$ ), which must all converge to cause a similar degree of functional protection of the permeability transition pore complex (the end effector), phosphorylate and inhibit the activity of GSK-3 $\beta$, which we have found serves as a point of integration - a master switch - immediately proximal to the permeability transition pore complex. We conclude that the general mechanism of protection is the convergence of these pathways via inhibition of GSK-3 $\beta$ on the end effector, the permeability transition pore complex, to limit MPT induction.

Since we demonstrated that there is a direct correlation between cell survival and the ability to prevent MPT induction, the MPT susceptibility can be interpreted as a marker or proxy for the size of the infarct resulting from arterial occlusion (for a period less than that causing a completed infarction). The present experiments using 16 different proven cardio/neuroprotective agents reinforce the conclusion that the ability to protect the permeability transition pore complex from MPT induction is directly predictive of the ability to reduce infarct size. (These results have also been extended to include erythropoietin, $\mathrm{M}_{2}$ muscarinic, and $\alpha$ - and $\beta$-adrenergic stimulation; see Supplement 4).

Unresolved issues related to $P K C$ and the mito $K_{A T P}$. There remains an important unresolved issue concerning how $\mathrm{PKC}$ is related to mito $_{\text {ATP }}$ function and activation. $\mathrm{PKC}$ is certainly activated downstream of the mitoK $\mathrm{K}_{\mathrm{ATP}}$, based on the ability of $\mathrm{Dz}$ to induce (and of 5HD to block) PKC translocation. But PKC also appears to act upstream of the mitoK $\mathrm{K}_{\mathrm{ATP}}$, since PMA and CCPA $\left(\mathrm{A}_{1}\right)$ can induce protection (and PKC translocation; Figure 3, C and D) that can be blocked by 5HD (Figures 3A and 6D). 
The analysis of cellular flavoprotein fluorescence (serving as an index of mitoK $_{\text {ATP }}$ activity; ref. 46; see Figure 4B and Supplement 3 ) suggests that the mitoK $\mathrm{KTP}_{\mathrm{AT}}$ itself is a PKC target and, by inference, should have a PKC-binding site or receptor for activated C kinase (RACK) (47). Thus, it is plausible that the mito $K_{A T P}$ is playing more than one essential role in $\mathrm{PC}$ in these experiments (and that these are in a sequential loop). One role is the assumed upstream mechanism where it acts as a $\mathrm{K}^{+}$channel resulting in mitochondrial swelling, increasing respiration, ROS production, and PKC activation. We hypothesize that the other role is played out further downstream (which can even appear to be upstream, if $\mathrm{PKC}$ is activated as the triggering step, such as by PMA or CCPA), whereby this activated PKC comes back and binds to the mito$\mathrm{K}_{\mathrm{ATP}}$, where it is constrained to act locally as a kinase (Figure 10). This inference is supported by the fact that $5 \mathrm{HD}$ can block the protection of PMA, Hoe, DADLE, and CCPA, which do not share the capacity of $\mathrm{Dz}$ by itself to activate a flavoprotein response but which do manage to alter the flavoprotein response to $\mathrm{Dz}$ (Supplement 3). We also speculate that the characteristic memory of PC is "encoded" by the physical mitochondrial swelling, and that its persistence during mito $\mathrm{K}_{\mathrm{ATP}}$ activation is maintained through the apparent feedback amplification on $\mathrm{K}^{+}$conductance by PKC.

Although the mito $K_{A T P}$ has not been identified, we propose that the ability to serve as a RACK, in the presence of a complex with GSK-3 $\beta$ (and certain elements regulating the susceptibility of the permeability transition pore to undergo the MPT, such as Bcl-2), may be an integral part of the mechanism by which the mitoK $\mathrm{K}_{\mathrm{ATP}}$ works in PC. Furthermore, in this model 5HD would act as an inhibitor by preventing the binding of PKC to the mito $_{\text {ATP }}$ RACK site (in addition to blocking the activation of the $\mathrm{K}^{+}$conductance). Proof of these predicted mechanisms will require further testing.

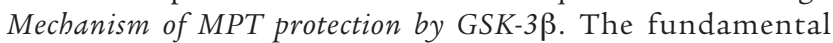
finding of this work is that a comprehensive survey of distinct mechanisms demonstrated that protection signaling integrates through GSK-3 $\beta$, which in turn protects the end effector, the permeability transition pore complex, from MPT induction during periods of oxidant stress. To exclude the possibility of functional redundancy by GSK-3 $\alpha$, we used RNA interference to specifically inhibit each GSK-3 isoform, as well as TG mice with cardiac-specific expression of a constitutively active (signal-resistant) form of GSK-3 (GSK-3 $\beta$ S9A) (24); these experiments confirmed the unique role of GSK-3 $\beta$ in cell protection.

However, the exact permeability transition pore-regulatory target(s) of GSK-3 $\beta$ remains uncertain. Searching, with Scansite (48), for motifs within proteins (in the Swiss-Prot database; http:// us.expasy.org/sprot/) (49) that are likely to be phosphorylated by the serine/threonine kinase GSK-3 $\beta$ yielded several candidate permeability transition pore-regulatory targets: $\mathrm{Bcl}-2$, the Bcl-2-binding protein Bis (also called Bcl-2-binding athanogene-3 [BAG-3]) (50), and the serine/threonine protein phosphatase $2 \mathrm{~A}$ (PP2A, subunit B). Phosphorylation of serine-70, serine- 87 , or threonine- 69 of $\mathrm{Bcl}-2$, each of which is a candidate motif of GSK-3 $\beta$, represents an inactivation signal that can confer a loss of antiapoptotic influence $(51,52)$. Since GSK-3 $\beta$ is an active kinase in the basal state, its inhibition during protection signaling could reduce the background level of $\mathrm{Bcl}-2$ phosphorylation at these regulatory sites and unmask enhanced $\mathrm{Bcl}-2$ function. It is plausible that GSK-3 $\beta$ regulation of PP2A at the effector complex site could participate at this level as well. Furthermore, the Bcl-2-binding protein Bis, which is synergistic with Bcl-2 in preventing cell death, is expressed at high levels in heart and skeletal muscle, which has led to the speculation that Bis may be important for the survival of these long-lived cells (50).

$\mathrm{Bcl}-2$ can protect the heart against ischemia/reperfusion injury $(53,54)$. The BH4 domain of Bcl-xL has been shown to be sufficient for protection against mitochondrial dysfunction and apoptosis (55), and hearts perfused with a peptide corresponding to residues 4-23 of $\mathrm{Bcl}-\mathrm{xL}$ conjugated to the protein transduction domain of HIV TAT (TAT-BH4) demonstrated reduced injury after ischemia/reperfusion as manifested by an approximately $18 \%$ reduction in infarct size (56). In the present model, TAT-BH4 enhanced the MPT threshold to ROS by about $40 \%$ in cardiac myocytes, consistent with the infarction data (D.B. Zorov et al., unpublished observations).

We have demonstrated that each of the separate protection agents used here converges on GSK-3 $\beta$, which results in the enhancement of the MPT ROS threshold, but it remains unresolved how this GSK- $3 \beta$ signal specifically provides this protection. While we have initial evidence supporting an important role for Bcl-2-family proteins in this protection-signaling cascade, we cannot exclude a role for some common alteration in the capacity to scavenge or produce ROS as a downstream mediator of GSK-3 $\beta$ signaling that in turn results in this enhanced MPT ROS threshold. Further investigations of these mechanisms are required.

Mechanistic implications of protection signaling. Ischemic PC has been shown to be a clinically relevant phenomenon affording significant protection in humans (3). Thus, in certain populations with an increased incidence and prevalence of cardiovascular disease, treatments that may unexpectedly interfere with natural PC mechanisms (which would be triggered during transient premonitory episodes of ischemia, such as angina) could increase morbidity and mortality. Sulfonylureas are among the most widely used pharmaceuticals in the treatment of type 2 diabetes mellitus but have been associated with an unexpected and unexplained small increase in cardiovascular mortality in large epidemiology studies $(57,58)$. Certain sulfonylureas are known to inhibit the mitoK $\mathrm{K}_{\mathrm{ATP}}$, and we have found that glibenclamide (the prototype first-generation sulfonylurea) can abolish the MPT protection of Dz as effectively as 5HD can (S.-H. Kim and S.J. Sollott, unpublished observations). Similarly, while it is plausible that free radical mechanisms are involved in the pathogenesis of aging, atherosclerosis, and cancer, large epidemiology studies have not clearly established the benefits of antioxidants (e.g., ref. 59). The present experiments demonstrate that $\mathrm{PC}$ afforded by the entire mitochondrial-sweller class (including hypoxic PC) is prevented by ROS scavengers.

We found that PC triggers cause an increase in FFA $\beta$-oxidation and respiration, which are essential steps in the PC mechanism. That leptin is able to trigger PC and completely reproduce MPT protection, and that PFAO inhibition is able to block the MPT protection afforded by all mitochondrial swellers, prove this. Thus, the clinical use of PFAO inhibitors to treat angina, while effective for symptomatic control (60), may yet prove to be complicated by the parallel loss of endogenous protection mechanisms. It is plausible that at least some of the benefits of treating diabetes with sulfonylureas, or treating atherosclerosis with antioxidants or PFAO inhibitors, could be offset when the endogenous MPT protection mechanisms are unintentionally inhibited, especially in populations at increased risk of vascular disease, cancer, or age-related degeneration.

On the other hand, in certain human populations in which the endogenous capacity for protection signaling has become 
impaired or lost, such as during aging $(61,62)$ or, potentially, in those who are taking sulfonylureas, antioxidants, or PFAO inhibitors, engagement of MPT-protective signaling mechanisms that do not require mitochondrial swelling may still be effective. Specifically, signaling through insulin and IGF-1, erythropoietin, GLP-1, $\mathrm{Li}^{+}$, and SB (216763 and 415286) may still be effective under these conditions. The past empiric use of infusions of glucose-insulin- $\mathrm{K}^{+}(\mathrm{GIK})$ in treatment of myocardial infarction may now become recommended for its possible direct cardioprotective effect. Because aging may be a special case in which there are multiple (partial) defects in upstream signaling mechanisms, $\mathrm{Li}^{+}$and/ or SB (216763 and 415286) may be particularly effective, since they would bypass these upstream mechanisms and act directly on the downstream protection-effector kinase, GSK-3 $\beta$ (18). Thus, it might be reasonable to consider adding $\mathrm{Li}^{+}$(or another GSK-3 inhibitor) to GIK in the treatment of acute ischemic syndromes, myocardial infarction, and stroke.

1. Green, D.R., and Reed, J.C. 1998. Mitochondria and apoptosis. Science. 281:1309-1312.

2. Murry, C.E., Jennings, R.B., and Reimer, K.A. 1986. Preconditioning with ischemia: a delay of lethal cell injury in ischemic myocardium. Circulation. 74:1124-1136.

3. Kloner, R.A., Bolli, R., Marban, E., Reinlib, L., and Braunwald, E. 1998. Medical and cellular implications of stunning, hibernation, and preconditioning: an NHLBI workshop. Circulation. 97:1848-1867.

4. Inoue, I., Nagase, H., Kishi, K., and Higuti, T. 1991. ATP-sensitive $\mathrm{K}+$ channel in the mitochondrial inner membrane. Nature. 352:244-247.

5. Garlid, K.D., et al. 1997. Cardioprotective effect of diazoxide and its interaction with mitochondrial ATP-sensitive $\mathrm{K}+$ channels. Possible mechanism of cardioprotection. Circ. Res. 81:1072-1082.

6. Gross, G.J. 2000. The role of mitochondrial KATP channels in cardioprotection. Basic Res. Cardiol. 95:280-284.

7. Kloner, R.A., and Jennings, R.B. 2001. Consequences of brief ischemia: stunning, preconditioning, and their clinical implications: part 2. Circulation. 104:3158-3167.

8. Schulz, R., Cohen, M.V., Behrends, M., Downey, J.M., and Heusch, G. 2001. Signal transduction of ischemic preconditioning. Cardiovasc. Res. 52:181-198.

9. Akao, M., Teshima, Y., and Marban, E. 2002. Antiapoptotic effect of nicorandil mediated by mitochondrial ATP-sensitive potassium channels in cultured cardiac myocytes. J. Am. Coll. Cardiol. 40:803-810.

10. Hunter, D.R., and Haworth, R.A. 1979. The Ca2+induced membrane transition in mitochondria. I. The protective mechanisms. Arch. Biochem. Biophys. 195:453-459.

11. Crompton, M., and Costi, A. 1990. A heart mitochondrial $\mathrm{Ca} 2+$-dependent pore of possible relevance to re-perfusion-induced injury. Evidence that ADP facilitates pore interconversion between the closed and open states. Biochem. J. 266:33-39.

12. Halestrap, A.P., and Brenner, C. 2003. The adenine nucleotide translocase: a central component of the mitochondrial permeability transition pore and key player in cell death. Curr. Med. Chem. 10:1507-1525

13. Javadov, S.A., et al. 2003. Ischaemic preconditioning inhibits opening of mitochondrial permeability transition pores in the reperfused rat heart. J. Physiol. 549:513-524.

14. Hausenloy, D.J., Maddock, H.L., Baxter, G.F., and Yellon, D.M. 2002. Inhibiting mitochondrial permeability transition pore opening: a new paradigm

\section{Acknowledgments}

We thank H.A. Spurgeon, A.A. Starkov, and E.G. Lakatta for useful discussions, and A. Chesley and M.T. Crow for help with GSK-3 gene silencing via RNA interference. This work was supported by the Intramural Research Program, National Institute on Aging, NIH.

Received for publication August 28, 2003, and accepted in revised form March 30, 2004.

Address correspondence to: Steven J. Sollott, Laboratory of Cardiovascular Science, Gerontology Research Center, Box 13, Intramural Research Program, National Institute on Aging, 5600 Nathan Shock Drive, Baltimore, Maryland 21224-6825, USA. Phone: (410) 558-8657; Fax: (410) 558-8150; E-mail: sollotts@grc.nia.nih.gov.

Magdalena Juhaszova, Dmitry B. Zorov, and Suhn-Hee Kim contributed equally to this work.

for myocardial preconditioning? Cardiovasc. Res. 55:534-543.

15. Korge, P., Honda, H.M., and Weiss, J.N. 2002. Protection of cardiac mitochondria by diazoxide and protein kinase $\mathrm{C}$ : implications for ischemic preconditioning. Proc. Natl. Acad. Sci. U. S. A. 99:3312-3317.

16. Akao, M., et al. 2003. Differential actions of cardioprotective agents on the mitochondrial death pathway. Circ. Res. 92:195-202.

17. Zorov, D.B., Filburn, C.R., Klotz, L.O., Zweier, J.L., and Sollott, S.J. 2000. Reactive oxygen species (ROS)-induced ROS release: a new phenomenon accompanying induction of the mitochondrial permeability transition in cardiac myocytes. J. Exp. Med. 192:1001-1014.

18. Tong, H., Imahashi, K., Steenbergen, C., and Murphy, E. 2002. Phosphorylation of glycogen synthase kinase-3beta during preconditioning through a phosphatidylinositol-3-kinase-dependent pathway is cardioprotective. Circ. Res. 90:377-379.

19. Woodgett, J.R. 1990. Molecular cloning and expression of glycogen synthase kinase-3/factor A. EMBOJ. 9:2431-2438

20. Plyte, S.E., Hughes, K., Nikolakaki, E., Pulverer, B.J., and Woodgett, J.R. 1992. Glycogen synthase kinase-3: functions in oncogenesis and development. Biochim. Biophys. Acta. 1114:147-162.

21. Hoeflich, K.P., et al. 2000. Requirement for glycogen synthase kinase-3beta in cell survival and NF-kappaB activation. Nature. 406:86-90.

22. Cohen, P., and Frame, S. 2001. The renaissance of GSK3. Nat. Rev. Mol. Cell Biol. 2:769-776.

23. Cross, D.A., Alessi, D.R., Cohen, P., Andjelkovich, M., and Hemmings, B.A. 1995. Inhibition of glycogen synthase kinase- 3 by insulin mediated by protein kinase B. Nature. 378:785-789.

24. Antos, C.L., et al. 2002. Activated glycogen synthase- 3 beta suppresses cardiac hypertrophy in vivo. Proc. Natl. Acad. Sci. U. S. A. 99:907-912.

25. Capogrossi, M.C., Kort, A.A., Spurgeon, H.A., and Lakatta, E.G. 1986. Single adult rabbit and rat cardiac myocytes retain the $\mathrm{Ca} 2+-$ and species-dependent systolic and diastolic contractile properties of intact muscle. J. Gen. Physiol. 88:589-613.

26. Chesley, A., et al. 2000. The beta(2)-adrenergic receptor delivers an antiapoptotic signal to cardiac myocytes through $\mathrm{G}(\mathrm{i})$-dependent coupling to phosphatidylinositol $3^{\prime}$-kinase. Circ. Res. 87:1172-1179

27. Vila Petroff, M.G., et al. 2001. Endogenous nitric oxide mechanisms mediate the stretch dependence of $\mathrm{Ca} 2+$ release in cardiomyocytes. Nat. Cell Biol. 3:867-873.

28. Mannella, C.A., and Parsons, D.P. 1977. Small- angle X-ray scattering from mitochondria. Biochim. Biophys. Acta. 470:242-250.

29. Aon, M.A., Cortassa, S., Marban, E., and O'Rourke, B. 2003. Synchronized whole cell oscillations in mitochondrial metabolism triggered by a local release of reactive oxygen species in cardiac myocytes. J. Biol. Chem. 278:44735-44744.

30. Chen, L., et al. 2001. Opposing cardioprotective actions and parallel hypertrophic effects of delta PKC and epsilon PKC. Proc. Natl. Acad. Sci. U. S. A. 98:11114-11119.

31. Pain, T., et al. 2000. Opening of mitochondrial $\mathrm{K}(\mathrm{ATP})$ channels triggers the preconditioned state by generating free radicals. Circ. Res. 87:460-466.

32. Chance, B., Sies, H., and Boveris, A. 1979. Hydroperoxide metabolism in mammalian organs. Physiol. Rev. 59:527-605.

33. Dos Santos, P., et al. 2002. Mechanisms by which opening the mitochondrial ATP- sensitive $\mathrm{K}+$ channel protects the ischemic heart. Am. J. Physiol. 283: H284-H295.

34. Vila Petroff, M.G., Egan, J.M., Wang, X., and Sollott, S.J. 2001. Glucagon-like peptide-1 increases cAMP but fails to augment contraction in adult rat cardiac myocytes. Circ. Res. 89:445-452.

35. Kowaltowski, A.J., Castilho, R.F., and Vercesi, A.E. 2001. Mitochondrial permeability transition and oxidative stress. FEBS Lett. 495:12-15.

36. Halestrap, A.P. 1989. The regulation of the matrix volume of mammalian mitochondria in vivo and in vitro and its role in the control of mitochondrial metabolism. Biochim. Biophys. Acta. 973:355-382.

37. Atkinson, L.L., Fischer, M.A., and Lopaschuk, G.D. 2002. Leptin activates cardiac fatty acid oxidation independent of changes in the AMP-activated protein kinase-acetyl-CoA carboxylase-malonyl-CoA axis. J. Biol. Chem. 277:29424-29430.

38. Minokoshi, Y., et al. 2002. Leptin stimulates fattyacid oxidation by activating AMP-activated protein kinase. Nature. 415:339-343.

39. Stambolic, L., Ruel, V., and Woodgett, J.R. 1996. Lithium inhibits glycogen synthase kinase-3 activity and mimics wingless signalling in intact cells. Curr. Biol. 6:1664-1668.

40. Cross, D.A., et al. 2001. Selective small-molecule inhibitors of glycogen synthase kinase-3 activity protect primary neurones from death. J. Neurochem. 77:94-102.

41. Bijur, G.N., and Jope, R.S. 2003. Rapid accumulation of Akt in mitochondria following phosphatidylinositol 3-kinase activation. J. Neurochem. 87:1427-1435.

42. Dajani, R., et al. 2001. Crystal structure of glycogen synthase kinase 3 beta: structural basis for phosphate-primed substrate specificity and autoinhibi- 
tion. Cell. 105:721-732.

43. Cave, A.C., Collis, C.S., Downey, J.M., and Hearse, D.J. 1993. Improved functional recovery by ischaemic preconditioning is not mediated by adenosine in the globally ischaemic isolated rat heart. Cardiovasc. Res. 27:663-668.

44. Ganote, C.E., Armstrong, S., and Downey, J.M. 1993. Adenosine and A1 selective agonists offer minimal protection against ischaemic injury to isolated rat cardiomyocytes. Cardiovasc. Res. 27:1670-1676.

45. Armstrong, S., Downey, J.M., and Ganote, C.E. 1994. Preconditioning of isolated rabbit cardiomyocytes: induction by metabolic stress and blockade by the adenosine antagonist SPT and calphostin C, a protein kinase C inhibitor. Cardiovasc. Res. 28:72-77.

46. Sato, T., O’Rourke, B., and Marban, E. 1998. Modulation of mitochondrial ATP-dependent K+ channels by protein kinase C. Circ. Res. 83:110-114.

47. Mochly-Rosen, D. 1995. Localization of protein kinases by anchoring proteins: a theme in signal transduction. Science. 268:247-251.

48. Yaffe, M.B., et al. 2001. A motif-based profile scanning approach for genome-wide prediction of signaling pathways. Nat. Biotechnol. 19:348-353.

49. Boeckmann, B., et al. 2003. The SWISS-PROT pro- tein knowledgebase and its supplement TrEMBL in 2003. Nucleic Acids Res. 31:365-370.

50. Lee, J.H., et al. 1999. Bis, a Bcl-2-binding protein that synergizes with $\mathrm{Bcl}-2$ in preventing cell death. Oncogene. 18:6183-6190.

51. Ojala, P.M., et al. 2000. The apoptotic v-cyclinCDK6 complex phosphorylates and inactivates Bcl-2. Nat. Cell Biol. 2:819-825.

52. Haldar, S., Jena, N., and Croce, C.M. 1995. Inactivation of Bcl-2 by phosphorylation. Proc. Natl. Acad. Sci. U. S. A. 92:4507-4511.

53. Chen, Z., Chua, C.C., Ho, Y.S., Hamdy, R.C., and Chua, B.H. 2001. Overexpression of Bcl-2 attenuates apoptosis and protects against myocardial I/R injury in transgenic mice. Am. J. Physiol. 280:H2313-H2320.

54. Brocheriou, V., et al. 2000. Cardiac function improvement by a human $\mathrm{Bcl}-2$ transgene in a mouse model of ischemia/reperfusion injury. J. Gene Med. 2:326-333.

55. Shimizu, S., Konishi, A., Kodama, T., and Tsujimoto, Y. 2000. BH4 domain of antiapoptotic Bcl-2 family members closes voltage-dependent anion channel and inhibits apoptotic mitochondrial changes and cell death. Proc. Natl. Acad. Sci. U. S. A.
97:3100-3105.

56. Chen, M., Won, D.J., Krajewski, S., and Gottlieb, R.A. 2002. Calpain and mitochondria in ischemia/ reperfusion injury. J. Biol. Chem. 277:29181-29186.

57. Garratt, K.N., et al. 1999. Sulfonylurea drugs increase early mortality in patients with diabetes mellitus after direct angioplasty for acute myocardial infarction. J. Am. Coll. Cardiol. 33:119-124.

58. Scognamiglio, R., et al. 2002. Effects of treatment with sulfonylurea drugs or insulin on ischemiainduced myocardial dysfunction in type 2 diabetes. Diabetes. 51:808-812.

59. Heart Protection Study Collaborative Group. 2002. MRC/BHF Heart Protection Study of antioxidant vitamin supplementation in 20536 high-risk individuals: a randomised placebo-controlled trial. Lancet. 360:23-33.

60. Stanley, W.C. 2002. Partial fatty acid oxidation inhibitors for stable angina. Expert Opin. Investig. Drugs. 11:615-629.

61. Abete, P., et al. 1996. Preconditioning does not prevent postischemic dysfunction in aging heart. J. Am. Coll. Cardiol. 27:1777-1786.

62. Wallace, D.C., and Melov, S. 1998. Radicals r'aging. Nat. Genet. 19:105-106. 